\title{
Discontinuities and hysteresis in quantized average consensus *
}

\author{
Francesca Ceragioli $\quad$ Claudio De Persis ${ }^{\ddagger} \quad$ Paolo Frasca ${ }^{\S}$
}

October 29, 2018

\begin{abstract}
We consider continuous-time average consensus dynamics in which the agents' states are communicated through uniform quantizers. Solutions to the resulting system are defined in the Krasowskii sense and are proven to converge to conditions of "practical consensus". To cope with undesired chattering phenomena we introduce a hysteretic quantizer, and we study the convergence properties of the resulting dynamics by a hybrid system approach.
\end{abstract}

\section{Introduction}

Communication constraints play a major role in consensus and related problems of distributed computation and control. Such constraints can be represented by a graph of available communication links among agents, together with further restrictions on which information can be exchanged across links. Recently, the constraint of quantization, that is of communication restricted to a discrete set of symbols, has received significant attention. Although most works to-date have dealt with discrete-time dynamics, it is worth considering the same restrictions in the context of continuous-time dynamics, because the dynamics of the agents is naturally described by continuous-time systems in many applications. An example of this is robotic networks. One might argue that it is possible to study the effect of quantization on continuous-time systems by considering their discretized or sampled-data model. However, implementing consensus control algorithms by discretizing the dynamics requires implicitly that all the agents sample synchronously with the same clock, a requirement which is difficult to satisfy in practice. This lack of synchronicity may disrupt the convergence properties of the algorithm and thus asks for a different approach. This paper proposes an approach which deals with consensus problems in continuous-time without relying on sampled-data systems: consensus is achieved by quantized measurements which are transmitted asynchronously. Indeed, the quantization of the states induces a partition of

\footnotetext{
*An abridged version of this paper has been presented at the 8th IFAC Symposium on Nonlinear Control Systems, Bologna, Italy, September 2010. The work of the first and third author is partially supported by MIUR PRIN 2008 Sistemi distribuiti su larga scala: stima, ottimizzazione e controllo, con applicazioni. The work of the second author is partially supported by MIUR PRIN 2008 Advanced methods for feedback control of uncertain nonlinear systems, Progetto Ricercatori AST 2009 and a Johns Hopkins University Applied Physics Laboratory grant.

${ }^{\dagger}$ Dipartimento di Matematica, Politecnico di Torino, Torino, Italy. francesca.ceragioli@polito.it.

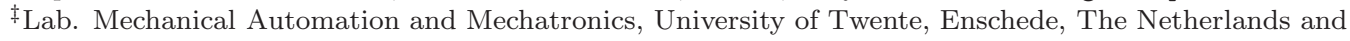
Dip. Informatica e Sistemistica, Sapienza Università di Roma, Roma, Italy. C.DePersis@ctw.utwente.nl.

${ }^{\S}$ Dipartimento di Matematica, Politecnico di Torino, Torino, Italy. paolo.frasca@polito.it.
} 
the space into regions and each agent transmits quantized information about its state only when the state crosses the boundary of the quantization regions. In this sense communication among the agents takes place asynchronously. Throughout the paper no assumption is imposed on the resolution of the quantizers, which therefore can be also very coarse. Hence, this paper specifically aims at giving a rigorous treatment of continuous-time average consensus dynamics with uniform quantization in communications. It is an established fact that consensus problems can be reformulated in terms of feedback control systems: as expected, when quantization enters the loop, the stabilization problem becomes more challenging. From a mathematical point of view, a consequence of quantization is that we obtain a system with a discontinuous righthand side, which is not guaranteed to admit solutions in classical sense. This paper proves that classical or Carathéodory solutions may in fact not exist: considering solutions in some generalized sense is thus unavoidable.

The literature provides different approaches to the technical problem of having systems with discontinuous righthand sides (see $[8,15]$ for a review of these topics). Tools from the theory of discontinuous differential equations and nonsmooth analysis have already been applied in consensus problems in [7,9]. Here we focus on Krasowskii solutions essentially for two reasons: effectiveness and generality. With regard to effectiveness, there are many results available concerning the existence and continuation of Krasowskii solutions, as well as a complete Lyapunov theory $[1,3]$. With respect to generality, since the set of Krasowskii solutions includes Filippov and Carathéodory solutions, results about Krasowskii solutions also hold for Filippov and Carathéodory solutions, provided that they exist. On the other hand, the set of Krasowskii solutions may be too large. In particular, from a practical point of view it may contain sliding modes which induce chattering phenomena. In the context of quantized consensus, chattering amounts to fast information transmission between the agents. This is undesirable because it results in algorithms which require large bandwidth communication channels to be implemented. To cope with this issue, we propose the use of a quantizer endowed with a hysteretic mechanism, and study the resulting dynamics by a hybrid system approach. Specifically, we provide an estimate of the data rate needed to implement the quantized continuous-time consensus algorithm. These results can be of interest to other application fields, including load balancing problems and real-time control systems.

With respect to earlier literature, our contribution is twofold. On one hand, we give a mathematical treatment, in terms of differential equations with discontinuous righthand sides, of a continuous-time consensus system under uniformly quantized communication of the states. We do this when the communication graph is only weakly connected and weight balanced. After showing basic properties of solutions, such as existence, boundedness and average preservation, we prove convergence to a set containing the equilibria of the system. This set depends on the communication graph, and is reduced to the set of equilibria in the particular case of symmetric graphs. On the other hand, our paper is the first to propose the application of hysteretic quantizers to solve a consensus problem with data rate constraints. Preliminarly, we prove that the system is well-posed, in the sense that a solution exists, is forward unique, and the set of switching times is locally finite. The main results consist in proving convergence and estimating the required data rate.

In our paper, we provide convergence results for both the Krasowskii and the hysteretic dynamics discussed above. Due to the constraint of static uniform quantization we cannot obtain exact consensus, but we can obtain approximations of the consensus condition which we informally refer to as "practical consensus". Similar conditions have been obtained elsewhere in the literature, which has already considered some related problems of quantized consensus. A number of publications have discussed -mostly in discrete-time systems- 
several options to deal with the quantization constraint, namely uniform deterministic quantizers [13, 18], uniform randomized quantizers [2], logarithmic quantizers [5], and adaptive quantizers [17]. Moreover, quantization has been considered also in gossip consensus algorithms $[16,6]$. Regarding continuous-time systems, relevant bibliography includes [7], which discusses discontinuous differential equations with consensus applications, and [22], which studies a rendezvous algorithm in which each agent tracks another agent assigned to it by a quantized control law. A recent paper ([11]) is also related: using graph-theoretical tools, the authors study a quantized consensus problem, under the assumption that the communication graph is a tree.

The present paper is organized as follows. In Section 2 we recall definitions and results from graph theory and about average consensus dynamics without quantization, recalling how the consensus problem can be reformulated in terms of a stability problem. We also present the problem of quantization in a continuous-time setting. In Section 3 we introduce state quantization and Krasowskii solutions and we study the fundamental properties of the system: we compute the set of equilibria, we prove average preservation, and we deduce asymptotic and finite-time convergence results. Then, in Section 4 we define and study the system under a hysteretic quantizer, in terms of a hybrid system. After proving that chattering can not occur, we study the fundamental properties of this system: equilibria, average preservation and convergence. Some simulations are given in Section 4.3 which illustrate our results and point to possible future research directions, which are discussed in our conclusions in Section 5 .

Notations. Given a subset $A$ of the Euclidean Space $\mathbb{R}^{N}$, we denote as $\bar{A}$ its topological closure, by int $(A)$ its interior and by $\partial A$ its boundary. Given $N \in \mathbb{N}$, we let $\mathbf{1}(\mathbf{0})$ be the $N \times 1$ vector whose entries are $1(0), I$ be the $N$-dimensional identity matrix and $\Omega=I-N^{-1} \mathbf{1} 1^{*}$, where the symbol ${ }^{*}$ denotes conjugate transpose. $\|\cdot\|$ denotes the Euclidean norm both for vectors and matrices.

\section{Preliminaries}

\subsection{Graph theory}

Let there be a weighted (directed) graph $G=(V, E, A)$, consisting of a node set $V=$ $\{1, \ldots, N\}$, an edge set $E \subset V \times V$ and an adjacency matrix $A \in \mathbb{R}_{\geq 0}^{N \times N}$ such that $A_{i j}>0$ if $(j, i) \in E$, and $A_{i j}=0$ if $(j, i) \notin E$. For every node, we define the set of its in-neighbors as $\mathcal{N}_{i}=\{j \in V:(j, i) \in E\}$. We assume no self-loops in the graph, that is $i \notin \mathcal{N}_{i}$ for every $i \in V$. Nodes (vertices) are referred to as agents, edges as links. Let $d_{\mathrm{in}, i}:=\sum_{j=1}^{n} A_{i j}$ and $d_{\text {out }, j}=\sum_{i=1}^{n} A_{i j}$ be, respectively, the in-degree and the out-degree of node $i \in V$. A graph is said to be weight-balanced if the out-degree of each node equals its in-degree. Let $D=\operatorname{diag}(A \mathbf{1})$ be the diagonal matrix whose diagonal entries are the in-degrees of each node, which are equal to the number of incoming edges if the nonzero entries of the adjacency matrix $A$ are all equal to 1 . Let $L=D-A$ be the Laplacian matrix of the graph $G$. Note that $L \mathbf{1}=\mathbf{0}$, and that $\mathbf{1}^{*} L=\mathbf{0}^{*}$ if and only if $G$ is weight-balanced. A path in a graph is an ordered list of edges. Given an edge $(i, j)$, we shall refer to $i$ and to $j$ as the tail and the head of the edge, respectively. An oriented path is an ordered list of edges such that the head of each edge is equal to the tail of the following one. The graph $G$ is said to be strongly connected if for any $i, j \in V$ there is an oriented path from $i$ to $j$ in $G$. Instead, it is said to be weakly connected if for each pair of nodes $i, j$ there exists a path which connects $i$ and $j$. Observe that weakly connected weight-balanced graphs are strongly connected graphs $[9$, Proposition 2]. Recall the following result, which can be derived from [4, Theorem 1.37] and 
[9, Formula (1) and Section 2.2].

Lemma 1 Let $G$ be a weighted graph and suppose it is weight-balanced and weakly connected. Let $L$ be its Laplacian matrix. Then:

(i) The matrix $\operatorname{Sym}(L):=\frac{L+L^{*}}{2}$ is positive semi-definite.

(ii) Denoted by $\lambda_{2}(\operatorname{Sym}(L))$ the smallest non-zero eigenvalue of $\operatorname{Sym}(L)$,

$$
x^{*} \operatorname{Sym}(L) x \geq \lambda_{2}(\operatorname{Sym}(L))\left\|x-\frac{\mathbf{1 1}^{*}}{N} x\right\|^{2},
$$

for all $x \in \mathbb{R}^{N}$, where $\|\cdot\|$ denotes the Euclidean norm.

Sometimes, it may be convenient to restrict our attention to symmetric graphs, that is graphs such that $A=A^{*}$. For a symmetric graph there is no distinction between strong and weak connectedness, so in that case we shall just say that the graph is connected.

\section{$2.2 \quad$ Feedback consensus dynamics}

Let $x: \mathbb{R}_{\geq 0} \rightarrow \mathbb{R}^{N}$ be a time dependent vector representing the agents' states. Its dynamics can be written in terms of the control system

$$
\dot{x}=u,
$$

where $x, u \in \mathbb{R}^{N}$. Our aim is to construct a control law $u: \mathbb{R} \rightarrow \mathbb{R}^{N}$ such that, for all initial conditions, solutions to (1) satisfy the average consensus condition, that is

$$
\lim _{t \rightarrow \infty} x(t)=x_{\text {ave }}(0) \mathbf{1},
$$

where we let $x_{\text {ave }}(t)=N^{-1} \mathbf{1}^{*} x(t)$. It turns out that one such control can be given in the feedback form $u=-L x$ so that the implemented system becomes

$$
\dot{x}(t)=-L x(t) .
$$

Componentwise, this reads as

$$
\dot{x}_{i}(t)=\sum_{j \in \mathcal{N}_{i}} A_{i j}\left(x_{j}(t)-x_{i}(t)\right), \quad \forall i \in V
$$

where we recall $\mathcal{N}_{i}$ is the set of the neighbors of agent $i$.

The following result, which can be deduced from [9], gives the weakest conditions for system (2) to converge to average consensus.

Lemma 2 If the weighted graph $G$ is weakly connected and weight-balanced, and $x(t)$ satisfies (2), then

$$
\lim _{t \rightarrow \infty} x(t)=x_{\text {ave }}(0) \mathbf{1}
$$

In view of the above result, from now on we shall make use of the following standing assumption, unless otherwise stated.

Assumption 1 The communication graph $G$ is weakly connected and weight-balanced. 


\subsection{Quantized consensus dynamics}

It is clear that the dynamics (2) is a rather idealized version of what can actually be implemented in a real control system. A very natural issue, as discussed in the introduction, is quantization. Let $q$ be a quantizer, that is a map $q: \mathbb{R} \rightarrow \mathcal{S}$ with $\mathcal{S}$ a discrete subset of $\mathbb{R}$ (If $z \in \mathbb{R}^{d}, q(z)$ is meant componentwise). Several quantized dynamics based on (2) are of interest from the point of view of the applications: indeed, quantization may be inherent either to communication or sensing among agents, or to the computation of the feedback control, or to its application to the system. In other words quantization can occur at the communication, sensor, computation or actuator level. If the sensed/communicated data about the neighbors states is quantized, due to the use of a digital lossless channel or to finite-precision sensors, the resulting dynamics is

$$
\dot{x}(t)=-L q(x(t)) .
$$

Despite its interest, the dynamics (4) has not received much attention yet, although its discrete-time counterpart has been widely studied, for instance in $[18,13]$. In the present paper, we shall focus on this dynamics, considering two different quantizers. First, we take a uniform static quantizer; then, we design a hysteretic quantizer which prevents chattering.

\section{Krasowskii quantized dynamics}

Let us consider a uniform quantizer $\mathrm{q}: \mathbb{R} \rightarrow \Delta \mathbb{Z}$, defined by

$$
\mathrm{q}(z)=\left\lfloor\frac{z}{\Delta}+\frac{1}{2}\right\rfloor \Delta .
$$

Note that $|\mathrm{q}(z)-z| \leq \frac{\Delta}{2}$. Moreover if $x \in \mathbb{R}^{N}$, we let $\mathrm{q}(x)=\left(\mathrm{q}\left(x_{1}\right), \ldots, \mathrm{q}\left(x_{N}\right)\right)^{*}$. We shall then consider the quantized dynamics

$$
\dot{x}=-L \mathrm{q}(x) .
$$

Since the righthand side of (5) is discontinuous, it is important to specify in which sense solutions have to be intended. One definition of solution is in the sense of Carathéodory : an absolutely continuous function $x(t)$ is a Carathéodory solution to (5) on an interval $I$ if it satisfies (5) for almost every $t \in I$. Such solution is said to be complete if $I=[0,+\infty)$. However, this natural definition is not suitable to study the system at hand, because of the following fact:

Proposition 1 (Carathéodory solutions) There are weakly connected and weight-balanced graphs and initial conditions for which no Carathéodory solution to (5) exists. Moreover, in these cases, there is a positive-measure set of initial conditions such that Carathéodory solutions starting from that set are not complete.

Proof: The righthand side of (5) is constant over open hypercubes with edge of length $\Delta$, and surfaces of discontinuity are hyperplanes. Each hyperplane is orthogonal to one of the elements of the canonical basis of $\mathbb{R}^{N}, e_{1}, \ldots, e_{N}$. Let us consider one such hyperplane, namely one which is orthogonal to $e_{j}$ and let us denote it by $S_{j}$. Let us consider a point $\hat{x} \in S_{j}$, i.e. such that $\hat{x}_{j}=\left(k+\frac{1}{2}\right) \Delta$ for some $k \in \mathbb{Z}$, and such that if $i \neq j$, then $\hat{x}_{i} \neq\left(h+\frac{1}{2}\right) \Delta$ for any $h \in \mathbb{Z}$. Let $I(\hat{x})$ be a neighborhood of $\hat{x}$ such that, for any $x \in I(\hat{x})$, it holds that $x_{j} \neq\left(h+\frac{1}{2}\right) \Delta$ for any integer $h \neq k$, and $x_{i} \neq\left(h+\frac{1}{2}\right) \Delta$ if $i \neq j$ for any $h \in \mathbb{Z}$. 
Let $I^{+}(\hat{x})=\left\{x \in I(\hat{x}): x_{j}>\left(k+\frac{1}{2}\right) \Delta\right\}$ and $I^{-}(\hat{x})=\left\{x \in I(\hat{x}): x_{j}<\left(k+\frac{1}{2}\right) \Delta\right\}$. Let $q$ be the value of $\mathrm{q}(x)$ in $I^{+}(\hat{x}), f^{+}$be the value of $-L \mathrm{q}(x)$ in $I^{+}(\hat{x})$ and $f^{-}$be the value of $-L \mathrm{q}(x)$ in $I^{-}(\hat{x})$. Then, letting the nonzero entries of the adjacency matrix to be all equal to 1 , the components $f^{+}$and $f^{-}$which are orthogonal to $S_{j}$ have simple expressions and interpretation:

$$
\begin{aligned}
& f_{\perp}^{+}:=\left\langle f^{+}, e_{j}\right\rangle=f_{j}^{+}=\sum_{k \in \mathcal{N}_{j}} q_{k}-d_{\mathrm{in}, j} q_{j}, \\
& f_{\perp}^{-}:=\left\langle f^{-}, e_{j}\right\rangle=f_{j}^{-}=f_{j}^{+}+d_{\mathrm{in}, j} \Delta,
\end{aligned}
$$

where $d_{\mathrm{in}, j}$ is the in-degree of node $j$. If either $f_{\perp}^{+}$is positive or $f_{\perp}^{+}<-d_{\mathrm{in}, j} \Delta$, then $f_{\perp}^{+}$ and $f_{\perp}^{-}$have the same sign, otherwise they have opposite sign. A numerical example for the latter case is as follows: let $d_{\mathrm{in}, j}=2, q_{j}=2 \Delta$ and $\left\{q_{k}: k \in \mathcal{N}_{j}\right\}=\{\Delta, 2 \Delta\}$, then $f_{j}^{+}=-\Delta$ and $f_{j}^{-}=\Delta$. In this case, $f_{\perp}^{+}<0$ and $f_{\perp}^{-}>0$, so that there are no Carathéodory solutions starting at $\hat{x}$. Moreover, solutions starting in a (sufficiently small) neighborhood of $\hat{x}$ reach the surface in finite time, and can not be extended further.

Example 1 Consider a graph with adjacency matrix whose entries are: $A_{12}=A_{21}=$ $1, A_{23}=A_{32}=1, A_{11}=A_{22}=A_{33}=A_{13}=A_{31}=0$ and the initial condition $\hat{x}=$ $\left(\Delta, \frac{3}{2} \Delta, 2 \Delta\right)$. Clearly the graph satisfies Assumption 1 . Note that the righthand side of (5) is discontinuous at $\hat{x}$, being on the plane $x_{2}=\frac{3}{2} \Delta$. Following the proof of Proposition 1 one can easily check that $f_{2}^{+}=-\Delta$ and $f_{2}^{-}=\Delta$. A possible Carathéodory solution issuing from $\hat{x}$ could not leave the surface $x_{2}=\frac{3}{2} \Delta$ in the direction of decreasing $x_{2}$ because $f_{2}^{-}=\Delta>0$ nor could leave the surface in the direction of increasing $x_{2}$, since $f_{2}^{+}=-\Delta<0$. The only possibility for a solution would be to remain on the surface but such a solution would not satisfy the definition of a Carathéodory solution. Hence, there are no Carathéodory solutions issuing from $\hat{x}$.

In view of Proposition 1, in the sequel of this paper we shall consider solutions in a more general sense, which is due to Krasowskii. An absolutely continuous function $x(t)$ is a Krasowskii solution to (5) on an interval $I$ if it satisfies at almost every $t \in I$ the differential inclusion

$$
\dot{x} \in \mathcal{K}(-L \mathrm{q}(x)),
$$

where

$$
\mathcal{K}(-L \mathrm{q}(x))=\bigcap_{\delta>0} \overline{\mathrm{CO}}(-L \mathrm{q}(B(x, \delta))),
$$

and $B(x, \delta)$ is the Euclidean ball of radius $\delta$ centered in $x$. The solution is said to be complete if $I=[0,+\infty)$. Note that, thanks to Theorem 1 in [20], we have

$$
\mathcal{K}(-L \mathrm{q}(x))=-L \mathcal{K}(\mathrm{q}(x)) \subseteq-L\left(\underset{i \in V}{\times \mathcal{K}}\left(\mathrm{q}\left(x_{i}\right)\right)=\underset{i \in V}{\times}\left(\sum_{j \in \mathcal{N}_{i}} A_{i j}\left(\mathcal{K}\left(\mathrm{q}\left(x_{j}\right)\right)-\mathcal{K}\left(\mathrm{q}\left(x_{i}\right)\right)\right)\right)\right.
$$

where $\mathrm{X}_{i}$ denotes the Cartesian product of the sets indexed by $i$. Representations of the map $\mathrm{q}$ and of the set-valued map $\mathcal{K} \mathrm{q}$ are given in Figure 1.

Lemma 3 (Krasowskii solutions) For any $x^{0} \in \mathbb{R}^{N}$ there exists a complete Krasowskii solution $x(t)$ to (5) such that $x(0)=x^{0}$. 

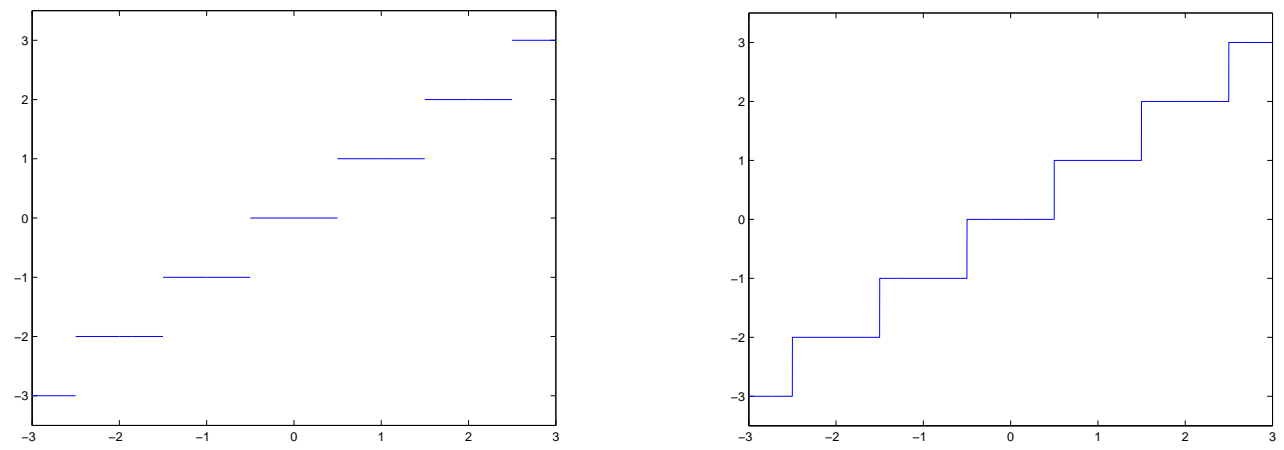

Figure 1: Visualization of the map $\mathrm{q}(x)$ and the set-valued map $\mathcal{K} \mathrm{q}(x)$, when $\Delta=1$.

Proof: Local existence of Krasowskii solutions is guaranteed by the fact that the righthand side of (5) is measurable and locally bounded (see [15]). By standard arguments completeness of solutions can be deduced by their boundedness (see, e.g., [19]). We then prove that solutions are bounded. Let $x(t)$ be a Krasowskii solution to (5) such that $x(0)=$ $x^{0}$. Let $m(t)=\min \left\{x_{i}(t), i \in V\right\}$ and $M(t)=\max \left\{x_{i}(t), i \in V\right\}$. Let $t_{0} \geq 0$ be fixed. We prove that $m(t) \geq m\left(t_{0}\right)$ for all $t \geq t_{0}$. Analogously it can be proven that $M(t) \leq M\left(t_{0}\right)$ for all $t \geq t_{0}$. By taking $t_{0}=0$ we will then deduce that for any $t>0$ and for any $i \in V$ one has $m(0) \leq x_{i}(t) \leq M(0)$, i.e. $x(t)$ is bounded. We first consider the case $m\left(t_{0}\right) \neq\left(k-\frac{1}{2}\right) \Delta$ for any $k \in \mathbb{Z}$. Assume by contradiction that there exists $\bar{t}>t_{0}$ such that $m(\bar{t})<m\left(t_{0}\right)$ and let $\bar{i} \in V$ be such that $x_{\bar{i}}(\bar{t})=m(\bar{t})$. Note that $m(t)$ is a continuous function since it is the minimum of a finite number of continuous functions. Then there exists $\delta>0$ such that $m(\bar{t}-\delta)=m\left(t_{0}\right)$ and for all $t \in(\bar{t}-\delta, \bar{t})$ one has $\mathrm{q}(m(t))=\mathrm{q}\left(x_{\bar{i}}(t)\right)=\mathrm{q}\left(m\left(t_{0}\right)\right)$ and $\mathrm{q}\left(x_{i}(t)\right) \geq \mathrm{q}\left(m\left(t_{0}\right)\right)$ for all $i \in V$. We remark that for all $t \in(\bar{t}-\delta, \bar{t})$ it holds $\mathcal{K}\left(\mathrm{q}\left(x_{\bar{i}}(t)\right)\right)=\left\{\mathrm{q}\left(m\left(t_{0}\right)\right)\right\}$ and $v \geq \mathrm{q}\left(m\left(t_{0}\right)\right)$ for all $v \in \mathcal{K}\left(\mathrm{q}\left(x_{j}(t)\right)\right)$ and any $j \in V$. Since $\dot{x}_{\bar{i}}(t) \in\left(-L \mathcal{K}(\mathrm{q}(x(t)))_{\bar{i}} \subseteq \sum_{j \in \mathcal{N}_{\bar{i}}} A_{i j}\left(\mathcal{K} \mathrm{q}\left(x_{j}(t)\right)-\mathcal{K} \mathrm{q}\left(x_{\bar{i}}(t)\right)\right)\right.$, we get that $\dot{x}_{\bar{i}}(t) \geq 0$ for almost all $t \in(\bar{t}-\delta, \bar{t})$. On the other hand, $x_{\bar{i}}(\bar{t})=m(\bar{t})<m\left(t_{0}\right)=m(\bar{t}-\delta) \leq x_{\bar{i}}(\bar{t}-\delta)$. Then there must exist a subset $I$ of $(\bar{t}-\delta, \bar{t})$ such that $I$ has positive measure and $\dot{x}_{\bar{i}}(t)<0$ for almost all $t \in I$, i.e. a contradiction. We now consider the case in which $m\left(t_{0}\right)=\left(k-\frac{1}{2}\right) \Delta$ for some $k \in \mathbb{Z}$. Let $t^{*}=\inf \left\{t>t_{0}: m(t)<m\left(t_{0}\right)\right\}$, and assume by contradiction that $t^{*}<+\infty$. Note that $m(t) \geq m\left(t_{0}\right)$ for $t \in\left[t_{0}, t^{*}\right)$ and, since $m$ is continuous, one also has $m\left(t^{*}\right)=m\left(t_{0}\right)$. Let us fix any $\bar{t}>t^{*}$ such that $\left(k-\frac{3}{2}\right) \Delta<m(t)<m\left(t_{0}\right)$ for any $t \in\left(t^{*}, \bar{t}\right)$. We take a sequence $\left\{t_{n}\right\}_{n \in \mathbb{N}}$ such that $t_{n} \in\left(t^{*}, \bar{t}\right)$ for any $n$ and $t_{n} \rightarrow t^{*}$. Since $m\left(t_{n}\right)<m\left(t_{0}\right)$, thanks to the previously analyzed case, we get that $m(t) \geq m\left(t_{n}\right)$ for all $t \geq t_{n}$. By passing to the limit we get that $m(t) \geq m\left(t^{*}\right)=m\left(t_{0}\right)$ for all $t \geq t^{*}$ and finally we have that $m(t) \geq m\left(t_{0}\right)$ for all $t \geq t_{0}$, again a contradiction.

We observe that Lemma 3 does not guarantee uniqueness of solutions, as explained in the following remark.

Remark 1 (Sliding mode) From the proof of Proposition 1, we see that if Carathéodory solutions originate from any point $\hat{x} \in S_{j}$ such that $f_{\perp}^{+}<0$ and $f_{\perp}^{-}>0$, then they do not exist, while Krasowkii solutions do. Krasowskii solutions starting in a neighborhood of $\hat{x}$ reach the surface in finite time and slide on it. We remark that such solutions are not 
backward unique. A general discussion about these behaviors can be found in [12, page 51].

Example 1 (Cont'd) Consider again the system in Example 1. It is straightforward to check that

$\mathcal{K}(-L \mathrm{q}(\hat{x}))=\overline{\mathrm{co}}\left\{\left(\begin{array}{r}\Delta \\ -\Delta \\ 0\end{array}\right),\left(\begin{array}{r}0 \\ \Delta \\ -\Delta\end{array}\right)\right\}=\left\{v \in \mathbb{R}^{3}: v=\left(\begin{array}{c}\lambda \Delta \\ (1-2 \lambda) \Delta \\ -(1-\lambda) \Delta\end{array}\right), \lambda \in[0,1]\right\}$.

Hence a Krasowskii solution issuing from $\hat{x}$ is the solution to $\dot{x}_{1}=\frac{\Delta}{2}, \dot{x}_{2}=0, \dot{x}_{3}=-\frac{\Delta}{2}$ with initial condition $\hat{x}$. On the other hand, as explained previously in Example 1, no Carathéodory solution issuing from $\hat{x}$ exists.

A preliminary result establishes that the average of the states is preserved by Krasowskii solutions to (5): this fact will be a key step to obtain many of the following results. We recall that this result holds under the standing Assumption 1: the same is true for all the following ones, with the exception of Theorem 2 and Proposition 4 which require a stronger assumption.

Lemma 4 (Average preservation) Let $x(t)$ be a Krasowskii solution to (5). Then, $x_{\text {ave }}(t)=$ $x_{\text {ave }}(0)$ for all $t \geq 0$.

Proof: By definition, $x_{\text {ave }}(t)=N^{-1} \mathbf{1}^{*} x(t)$, and then $\dot{x}_{\text {ave }}(t)=N^{-1} \mathbf{1}^{*} \dot{x}(t)$. By $(6)$,

$$
\dot{x}_{\text {ave }}(t) \in N^{-1} \mathbf{1}^{*} \mathcal{K}(-L \mathrm{q}(x(t))) \text { for a.e.t. }
$$

Thanks to the first equality in (7) and the fact that $\mathbf{1}^{*} L=\mathbf{0}^{*}$,

$$
\dot{x}_{\text {ave }}(t) \in-N^{-1} \mathbf{1}^{*} L \mathcal{K}(\mathrm{q}(x(t)))=\{0\},
$$

then we get that $\dot{x}_{\text {ave }}(t)=0$ for a.e. $t$ and finally that $x_{\text {ave }}(t)$ is constant.

\subsection{Graph-dependent convergence results}

A first set of results regards the limit behavior of system (5), which depends on the quantizer and the graph topology. The following proposition proves convergence of solutions to a certain set.

Theorem 1 (Convergence) If $x(t)$ is any Krasowskii solution to (5) and

$$
M=\left\{x \in \mathbb{R}^{N}: \frac{1}{\sqrt{N}}\left\|x-x_{\text {ave }}(0) \mathbf{1}\right\| \leq \frac{\|L\|}{\lambda_{2}(\operatorname{Sym}(L))} \frac{\Delta}{2}\right\},
$$

then $\operatorname{dist}(x(t), M) \rightarrow 0$ as $t \rightarrow+\infty$.

Proof: $\quad$ Let $y(t)=\Omega x(t)=x(t)-x_{\text {ave }}(t) \mathbf{1}$. Then, $\dot{y}=\Omega \dot{x} \in \Omega \mathcal{K}(-L \mathrm{q}(x))$. Since $\mathcal{K}(\Omega(-L \mathrm{q}(x)))=\Omega \mathcal{K}(-L \mathrm{q}(x))=\mathcal{K}(-L \mathrm{q}(x))=-L \mathcal{K} \mathrm{q}(x)$, we have $\dot{y} \in-L \mathcal{K} \mathrm{q}(x)$. Consider 
the function $V(y)=\frac{1}{2} y^{*} y$ and let $v \in \mathcal{K} \mathrm{q}(x)$. Note that if $v \in \mathcal{K} \mathrm{q}(x)$, then $\|v-x\| \leq \sqrt{N} \frac{\Delta}{2}$. We have that

$$
\begin{aligned}
\nabla V(y) \cdot \dot{y} & =-y^{*} L v \\
& =-y^{*} L(x+v-x) \\
& =-y^{*} L x-y^{*} L(v-x) \\
& =-y^{*} L y-y^{*} L(v-x) \\
& =-y^{*} \operatorname{Sym}(L) y-y^{*} L(v-x) \\
& \leq-\lambda_{2}(\operatorname{Sym}(L))\|y\|^{2}+\|y\|\|L\| \frac{\Delta}{2} \sqrt{N} \\
& =-\lambda_{2}(\operatorname{Sym}(L))\|y\|\left(\|y\|-\frac{\|L\|}{\lambda_{2}(\operatorname{Sym}(L))} \frac{\Delta}{2} \sqrt{N}\right),
\end{aligned}
$$

where the inequality in the second-last line follows from Lemma 1. This implies convergence to the set

$$
\left\{y \in \mathbb{R}^{N}:\|y\| \leq \frac{\|L\|}{\lambda_{2}(\operatorname{Sym}(L))} \frac{\Delta}{2} \sqrt{N}\right\} .
$$

Finally the statement follows from average preservation in Lemma 4.

With a slight extension of the argument leading to Theorem 1, we can prove finite-time convergence to a set larger than the set $M$ in Theorem 1, and provide an estimate of the convergence speed.

Corollary 1 (Finite-time convergence) If $x(t)$ is any Krasowskii solution to (5), then for any $\varepsilon \in(0,1)$ there exists a finite time $T(\varepsilon)$ such that $x(t)$ belongs to the set

$$
M(\varepsilon)=\left\{x \in \mathbb{R}^{N}: \frac{1}{\sqrt{N}}\left\|x-x_{\text {ave }}(0) \mathbf{1}\right\| \leq \frac{1}{1-\varepsilon} \frac{\|L\|}{\lambda_{2}(\operatorname{Sym}(L))} \frac{\Delta}{2}\right\} .
$$

for all $t \geq T(\varepsilon)$.

Proof: Consider, as in the proof of Theorem 1, the differential inclusion $\dot{y} \in-L K q(x)$, the function $V(y)=\frac{1}{2} y^{*} y$ and let $v \in \mathcal{K} \mathrm{q}(x)$. Then, as before,

$$
\nabla V(y) \cdot \dot{y} \leq-\lambda_{2}(\operatorname{Sym}(L))\|y\|\left(\|y\|-\frac{\|L\|}{\lambda_{2}(\operatorname{Sym}(L))} \frac{\Delta}{2} \sqrt{N}\right) .
$$

From the latter, we see that, if

$$
\|y(t)\|>\frac{1}{1-\varepsilon} \frac{\|L\|}{\lambda_{2}(\operatorname{Sym}(L))} \frac{\Delta}{2} \sqrt{N}
$$

then

$$
\begin{aligned}
\nabla V(y) \cdot \dot{y} & <-\varepsilon \lambda_{2}(\operatorname{Sym}(L))\|y\|^{2} \\
& =-2 \varepsilon \lambda_{2}(\operatorname{Sym}(L)) V(y)
\end{aligned}
$$

If at some time $t_{0}$ the condition (8) is satisfied by $y\left(t_{0}\right)$, then there exists $t>t_{0}$ such that the Lyapunov function computed along the trajectories of (5) satisfies

$$
V(y(t)) \leq \mathrm{e}^{\left.-2 \varepsilon \lambda_{2}(\operatorname{Sym}(L))\right)\left(t-t_{0}\right)} V\left(y\left(t_{0}\right)\right)
$$


and therefore

$$
\|y(t)\| \leq \mathrm{e}^{\left.-\varepsilon \lambda_{2}(\operatorname{Sym}(L))\right)\left(t-t_{0}\right)}\left\|y\left(t_{0}\right)\right\| .
$$

Assuming without loss of generality that $\left\|y\left(t_{0}\right)\right\| \neq 0$, from the latter inequality we conclude that, if at time $t_{0}$ the condition (8) is satisfied, then there exists a time

$$
T(\varepsilon)=\max \left\{0, \frac{-1}{\varepsilon \lambda_{2}(\operatorname{Sym}(L))} \ln \left(\frac{1}{1-\varepsilon} \frac{\|L\|}{\lambda_{2}(\operatorname{Sym}(L))} \frac{\Delta}{2} \frac{\sqrt{N}}{\left\|y\left(t_{0}\right)\right\|}\right)\right\}
$$

such that $\|y(t)\|$ satisfies

$$
\|y(t)\| \leq \frac{1}{1-\varepsilon} \frac{\|L\|}{\lambda_{2}(\operatorname{Sym}(L))} \frac{\Delta}{2} \sqrt{N},
$$

for any $t \geq t_{0}+T(\varepsilon)$. The thesis then follows, recalling the definition of $y(t)$ and Lemma 4 .

The above convergence results assert that the error induced by quantization, with respect to the non-quantized consensus dynamics (2), can be made arbitrarily small by decreasing the quantization error. Up to a $\sqrt{N}$ factor, due to the length of the vector, the committed error is proportional to $\frac{\|L\|}{\lambda_{2}}$, and then depends on the network. It is plain that it would be of interest to state a result of convergence to a stronger practical consensus condition, in which the committed error does not depend on the network topology but only on the quantizer precision. This issue is the topic of the next paragraph.

\subsection{Equilibria}

In this paragraph, we shall describe the equilibria of the system (5), which depend on the quantizer precision only. Hence, proving convergence to equilibria turns out to be a way to prove a practical consensus condition which does not depend on the network. The following proposition characterizes the equilibria of the system. We recall that $x_{0}$ is a (Krasowskii) equilibrium if the function $x(t) \equiv x_{0}$ is a (Krasowskii) solution, that is if $\mathbf{0} \in \mathcal{K}\left(-L \mathrm{q}\left(x_{0}\right)\right)$. Let

$$
\mathcal{D}=\left\{x \in \mathbb{R}^{N}: \exists k \in \mathbb{Z} \text { such that } \mathrm{q}\left(x_{i}\right)=\Delta k, \forall i \in V\right\} .
$$

Proposition 2 (Equilibria) The set of Krasowskii equilibria of (5) is $\overline{\mathcal{D}}$.

Proof: Note that $L \mathrm{q}(x)$ is zero in $\mathcal{D}$, and is discontinuous on the boundary of $\mathcal{D}$. Let us define the set of Krasowskii equilibria as $E=\left\{x \in \mathbb{R}^{N}: \mathbf{0} \in-L \mathcal{K}(\mathrm{q}(x))\right\}$, and let $\tilde{E}=\left\{x \in \mathbb{R}^{N}: \mathbf{0} \in-L\left(\times_{i} \mathcal{K}\left(\mathrm{q}\left(x_{i}\right)\right)\right)\right\}$, where we recall that $\times_{i}$ denotes the Cartesian product of the sets indexed by $i$. Since Theorem 1 in [20] implies that $\mathcal{K}(\mathrm{q}(x)) \subseteq \times_{i} \mathcal{K}\left(\mathrm{q}\left(x_{i}\right)\right)$, we have that $E \subseteq \tilde{E}$. In order to prove that $\mathcal{D}=E$, we will first prove that $\operatorname{int}(\mathcal{D}) \subset E$. Since $E$ is closed due to the fact that the set-valued map $\mathcal{K}(\mathrm{q}(\cdot))$ is upper semicontinuous (see, e.g., the definition of upper semi-continuity given in [10]), then also $\overline{\mathcal{D}} \subseteq E \subseteq \tilde{E}$. Later we will prove that $\tilde{E} \subseteq \overline{\mathcal{D}}$. These two facts imply that $\overline{\mathcal{D}}=E=\tilde{E}$, and namely our statement. Let us prove that $\mathcal{D} \subset E$. Let us assume $x_{0} \in \operatorname{int} \mathcal{D}$. Since $\mathrm{q}$ is continuous at $x_{0}$, then $\mathcal{K}\left(\mathrm{q}\left(x_{0}\right)\right)=\left\{\mathrm{q}\left(x_{0}\right)\right\}$ and $\mathcal{K}\left(-L \mathrm{q}\left(x_{0}\right)\right)=\left\{-L \mathrm{q}\left(x_{0}\right)\right\}=\{-L(\Delta k \mathbf{1})\}=\{-\Delta k L \mathbf{1}\}=\{\mathbf{0}\}$, i.e. $x_{0} \in E$. The points $x \in \overline{\mathcal{D}}$ also belong to $E$ thanks to the fact that $E$ is closed. Then, let us prove that $\tilde{E} \subseteq \overline{\mathcal{D}} . x_{0} \in \tilde{E}$ if there exists $v \in \times_{i} \mathcal{K}\left(\mathrm{q}\left(x_{0_{i}}\right)\right)$ such that $L v=0$. This is equivalent to the fact that $\operatorname{ker} L \cap \times{ }_{i} \mathcal{K}\left(\mathrm{q}\left(x_{0_{i}}\right)\right) \neq \emptyset$. Since $\operatorname{ker} L=\operatorname{span} \mathbf{1}$, there exists $v \in \operatorname{ker} L \cap \times{ }_{i} \mathcal{K}\left(\mathrm{q}\left(x_{0_{i}}\right)\right)$ if there exists $\lambda \in \mathbb{R}$ such that $v=\lambda \mathbf{1}$, i.e. $v_{i}=\lambda$ for any $i \in V$ and $v_{i} \in \mathcal{K}\left(\mathrm{q}\left(x_{0_{i}}\right)\right)$. Such $\lambda$ can be either $\lambda=\Delta k$ for some $k \in \mathbb{Z}$, or $\lambda \neq \Delta k$ for any $k \in \mathbb{Z}$. 
In the first case, for any $i \in V$ we have that $\left\{x_{i}: \lambda \in \mathcal{K}\left(\mathrm{q}\left(x_{i}\right)\right)\right\}=\left[\left(k-\frac{1}{2}\right) \Delta,\left(k+\frac{1}{2}\right) \Delta\right]$. In the second case, we have that $\left\{x_{i}: \lambda \in \mathcal{K}\left(\mathrm{q}\left(x_{i}\right)\right)\right\}=\left\{\left(\left\lfloor\frac{\lambda}{\Delta}\right\rfloor+\frac{1}{2}\right) \Delta\right\}$. Finally we get that if $v=\lambda \mathbf{1} \in \times{ }_{i} \mathcal{K}\left(\mathrm{q}\left(x_{0_{i}}\right)\right)$ then for every $i \in V$ either $x_{0_{i}} \in\left[\left(k-\frac{1}{2}\right) \Delta,\left(k+\frac{1}{2}\right) \Delta\right]$ for some $k \in \mathbb{Z}$, or $x_{0_{i}}=\left(k+\frac{1}{2}\right) \Delta$ for some $k \in \mathbb{Z}$, i.e. $x_{0} \in \overline{\mathcal{D}}$.

We now prove that $\overline{\mathcal{D}}$ is strongly invariant, i.e. there are no trajectories exiting $\overline{\mathcal{D}}$.

Proposition 3 (Strong invariance) If $x(t)$ is a Krasowskii solution to (5) such that $x(0) \in \overline{\mathcal{D}}$, then $x(t) \in \overline{\mathcal{D}}$ for all $t \geq 0$.

Proof: Recall that

$$
\overline{\mathcal{D}}=\left\{x \in \mathbb{R}^{N}: \exists k \in \mathbb{Z} \text { s. t. }\left(k-\frac{1}{2}\right) \Delta \leq x_{i} \leq\left(k+\frac{1}{2}\right) \Delta, \forall i \in V\right\} .
$$

Let $x(t)$ be a solution to (5) such that $x(0)=x^{0} \in \overline{\mathcal{D}}$. If $x^{0} \in \operatorname{int}(\mathcal{D})$, then $\operatorname{Lq}\left(x^{0}\right)=\mathbf{0}$, and thus $\mathcal{K} L \mathrm{q}\left(x^{0}\right)=\{\mathbf{0}\}$. Hence $\operatorname{int}(\mathcal{D})$ is invariant. Let then $x^{0} \in \partial \mathcal{D}$. Then there exist $k^{0} \in \mathbb{Z}$ and $V^{-}, V^{+} \subseteq V$ (not both empty) such that $x_{i}(0)=(k-1 / 2) \Delta$ for all $i \in V^{-}, x_{i}(0)=\left(k^{0}+1 / 2\right) \Delta$ for all $i \in V^{+}$, and $x_{i}(0) \in\left(\left(k^{0}-1 / 2\right) \Delta,\left(k^{0}+1 / 2\right) \Delta\right)$ for all $i \in V \backslash\left(V^{-} \cup V^{+}\right)$. Let us assume by contradiction that there exists $T>0$ such that $x(T) \notin \overline{\mathcal{D}}$, i.e. either there exists $\bar{i} \in V$ such that $x_{\bar{i}}(T)>\left(k^{0}+1 / 2\right) \Delta$ or there exists $\bar{j} \in V$ such that $x_{\bar{j}}(T)<\left(k^{0}-1 / 2\right) \Delta$. For brevity, we examine only the former case. Let $i^{*}$ be such that $x_{i^{*}}(T)=\max \left\{x_{i}(T), i \in V\right\}$. Without loss of generality, we can assume that $\left(k^{0}+3 / 2\right) \Delta>x_{i^{*}}(T)>\left(k^{0}+1 / 2\right) \Delta$ and $x_{i}(T)<\left(k^{0}+3 / 2\right) \Delta$ for all $i \in V$. Thanks to the continuity of $x(t)$, there exists $T^{\prime}<T$ such that $x_{i^{*}}\left(T^{\prime}\right)=\left(k^{0}+1 / 2\right) \Delta$ and $\left(k^{0}+1 / 2\right) \Delta<x_{i^{*}}(t)<\left(k^{0}+3 / 2\right) \Delta$ for all $t \in\left(T^{\prime}, T\right)$. Since $x_{i^{*}}(T)-x_{i^{*}}\left(T^{\prime}\right)>0$ there exists a subset $\mathcal{T}$ of $\left(T^{\prime}, T\right)$ such that $\mathcal{T}$ has positive Lebesgue measure and for all $t \in \mathcal{T}$ the derivative $\dot{x}_{i^{*}}(t)$ exists and is positive. On the other hand for all $t \in\left(T^{\prime}, T\right)$ one has $\left(k^{0}+1 / 2\right) \Delta<x_{i^{*}}(t)<\left(k^{0}+3 / 2\right) \Delta$, which implies $\mathrm{q}\left(x_{i^{*}}(t)\right)=\left(k^{0}+1\right) \Delta$ for all $t \in\left(T^{\prime}, T\right)$. Let us now consider any $v \in \mathcal{K}\left(\mathrm{q}(x(t))\right.$ with $t \in\left(T^{\prime}, T\right)$. It holds $v_{i^{*}}=\left(k^{0}+1\right) \Delta$ and $v_{j}<\left(k^{0}+1\right) \Delta$ for all $j \neq i^{*}$, and therefore $(-L v)_{i^{*}}<d_{\mathrm{in}, i^{*}}\left(k^{0}+1\right) \Delta-d_{\mathrm{in}, i^{*}}\left(k^{0}+1\right) \Delta=0$. From this fact it follows that $\dot{x}_{i^{*}}(t)<0$, i.e. a contradiction.

Next, we provide a second convergence result, stating that on any connected symmetric graph the quantized dynamics converges to the set of equilibria. This fact implies that the error induced by quantization does not depend on the network properties, but only on the quantizer.

Theorem 2 (Convergence to equilibria) If the weighted graph $G$ is symmetric and $x(t)$ is any Krasowskii solution to (5), then $\operatorname{dist}(x(t), \mathcal{D}) \rightarrow 0$ as $t \rightarrow \infty$.

Proof: We consider the function $V(x)=\frac{1}{2} x^{*} x$ and we prove that

$$
\nabla V(x) \cdot(-L v) \leq 0
$$


for every $x$ and for every $v \in \mathcal{K} \mathrm{q}(x)$. Let us first remark that $\mathcal{K} \mathrm{q}(x) \subseteq \times_{i} \mathcal{K}\left(\mathrm{q}\left(x_{i}\right)\right.$ and then $v \in \mathcal{K} \mathrm{q}(x)$ implies that $v \in \times{ }_{i} \mathcal{K}\left(\mathrm{q}\left(x_{i}\right)\right)$.

$$
\begin{aligned}
\nabla V(x) \cdot(-L v) & =-x^{*} L v \\
& =\sum_{i} x_{i} \sum_{j} A_{i j}\left(v_{j}-v_{i}\right) \\
& =-\frac{1}{2} \sum_{i j}\left(x_{j}-x_{i}\right) A_{i j}\left(v_{j}-v_{i}\right) \leq 0,
\end{aligned}
$$

where in the third equality we have used the symmetry of the graph, i.e. of the matrix $A$, and where the last inequality is a consequence of the fact that if $a, b \in \mathbb{R}, a \leq b$, then for all $u \in \mathcal{K} \mathrm{q}(a)$ and all $w \in \mathcal{K} \mathrm{q}(b)$ it holds $u \leq w$. Namely, all terms of the above summation are nonnegative. Let $Z=\left\{x \in \mathbb{R}^{N}: \exists v \in \mathcal{K} \mathrm{q}(x)\right.$ such that $\left.\nabla V(x) \cdot(-L v)=0\right\}$ and let $M$ be the largest weakly invariant subset of $\bar{Z}$. By LaSalle invariance principle for differential inclusions (see [3], Theorem 3) we have that any solution $x(t)$ to $\dot{x} \in-L(\mathcal{K}(\mathrm{q}(x))$ is such that $\operatorname{dist}(x(t), M) \rightarrow 0$ as $t \rightarrow+\infty$.

We now prove that $Z \subseteq \overline{\mathcal{D}}$. More precisely we prove that if $\bar{x} \notin \overline{\mathcal{D}}$ then $\bar{x} \notin Z$. Proving $\bar{x} \notin Z$ is equivalent to prove that for all $v \in \mathcal{K} \mathrm{q}(\bar{x})$ one has $\nabla V(\bar{x}) \cdot(-L v)<0$, i.e. $\sum_{i j}\left(\bar{x}_{j}-\bar{x}_{i}\right) A_{i j}\left(v_{j}-v_{i}\right)>0$, and, for this to hold, it is sufficient to prove that there exist $i, j$ such that $A_{i j} \neq 0$ and $\left(\bar{x}_{j}-\bar{x}_{i}\right)\left(v_{j}-v_{i}\right) \neq 0$. Let us then assume that $\bar{x} \notin \overline{\mathcal{D}}$.

This means that for all $k \in \mathbb{Z}$ there exists $\bar{i} \in V$ such that $\bar{x}_{\bar{i}}<(k-1 / 2) \Delta$ or there exists $\bar{j} \in V$ such that $\bar{x}_{\bar{j}}>(k+1 / 2) \Delta$. Let $k_{1}=\mathrm{q}\left(\bar{x}_{1}\right)$. Then there exists $i \in V$ (without loss generality we assume $i=2)$ such that $\bar{x}_{2}<\left(k_{1}-1 / 2\right) \Delta$ or $\bar{x}_{2}>\left(k_{1}+1 / 2\right) \Delta$. We consider the case $\bar{x}_{2}<\left(k_{1}-1 / 2\right) \Delta$. Let $k_{2}$ be such that $\mathrm{q}\left(\bar{x}_{2}\right)=k_{2} \Delta$. Clearly $k_{2}<k_{1}$. We examine the following possible cases:

(a) $\bar{x}_{1} \neq\left(k_{1}-1 / 2\right) \Delta$ and $\bar{x}_{2} \neq\left(k_{2}-1 / 2\right) \Delta$;

(b) $\bar{x}_{1}=\left(k_{1}-1 / 2\right) \Delta$ and $\bar{x}_{2} \neq\left(k_{2}-1 / 2\right) \Delta$;

(c) $\bar{x}_{1} \neq\left(k_{1}-1 / 2\right) \Delta$ and $\bar{x}_{2}=\left(k_{2}-1 / 2\right) \Delta$;

(d) $\bar{x}_{1}=\left(k_{1}-1 / 2\right) \Delta$ and $\bar{x}_{2}=\left(k_{2}-1 / 2\right) \Delta$.

In case (a) for all $v \in \mathcal{K} \mathrm{q}(\bar{x})$ one has $v_{1}=k_{1} \Delta$ and $v_{2}=k_{2} \Delta$ then $v_{1}-v_{2}=\Delta\left(k_{1}-k_{2}\right) \neq 0$ since $k_{1} \neq k_{2}$. In case (b) we remark that, since $\bar{x} \notin \overline{\mathcal{D}}$, then $k_{2} \leq k_{1}-2$. For all $v \in \mathcal{K} \mathrm{q}(\bar{x})$, one has $v_{1}=\alpha k_{1} \Delta+(1-\alpha)\left(k_{1}-1\right) \Delta=\Delta\left(k_{1}-1+\alpha\right)$ with $\alpha \in[0,1]$, and $v_{2}=k_{2} \Delta$. Then $v_{1}-v_{2}=\Delta\left(k_{1}-k_{2}-1+\alpha\right) \geq \Delta \neq 0$. Analogously in case (c) we get $v_{1}=\Delta k_{1}$, $v_{2}=\alpha\left(k_{2}-1\right) \Delta+(1-\alpha) k_{2} \Delta$, then $v_{1}-v_{2}=\Delta\left(k_{1}-k_{2}+\alpha\right)$ with $\alpha \in[0,1]$, then $v_{1}-v_{2} \neq 0$. Finally in case (d) we also have $k_{2} \leq k_{1}-2$, and moreover, since $\mathcal{K} \mathrm{q}(\bar{x}) \subseteq \times_{i} \mathcal{K}\left(\mathrm{q}\left(x_{i}\right)\right)$, for all $v \in \mathcal{K} \mathrm{q}(\bar{x})$ we have

$$
\begin{aligned}
& v_{1}=\Delta\left[\alpha_{1}\left(k_{1}-1\right)+\left(1-\alpha_{1}\right) k_{1}\right] \\
& v_{2}=\Delta\left[\alpha_{2}\left(k_{2}-1\right)+\left(1-\alpha_{2}\right) k_{2}\right],
\end{aligned}
$$

with $\alpha_{i} \in[0,1], i=1,2$. Then we get $v_{1}-v_{2}=\Delta\left[k_{1}-k_{2}-\alpha_{1}+\alpha_{2}\right] \neq 0$. Hence we have proved that $Z \subseteq \overline{\mathcal{D}}$. This fact also implies that $\bar{Z} \subseteq \overline{\mathcal{D}}$, and finally, $\operatorname{dist}(x(t), \overline{\mathcal{D}}) \rightarrow 0$ as $t \rightarrow+\infty$.

We remark that Theorem 2 does not imply that solutions converge to a point in $\mathcal{D}$. However, Lemma 4 implies that solutions whose initial conditions belong to the hyperplane $\sum_{i=1}^{N} x_{i}=N\left(k+\frac{1}{2}\right) \Delta$ for some $k \in \mathbb{Z}$ converge to the point $\left(k+\frac{1}{2}\right) \Delta \mathbf{1}$. 
One may ask whether solutions to $(5)$ reach $\overline{\mathcal{D}}$ in finite time. This claim is supported by numerical simulations, which we will illustrate in Section 4.3. Moreover, the following result shows that the claim is true for almost any initial condition.

Proposition 4 (Finite-time convergence to equilibria) If the weighted graph $G$ is symmetric and $x(t)$ is any Krasowskii solution to (5) such that $x_{\text {ave }}(0) \neq(k+1 / 2) \Delta$ for every $k \in \mathbb{Z}$, then there exists $T$ such that $x(T) \in \overline{\mathcal{D}}$.

Proof: Let us consider any Krasowskii solution $x(t)$ to $(5)$ such that $x(0) \notin \overline{\mathcal{D}}$ and its composition with the function $V(x)=\frac{1}{2} x^{*} x$. For almost all $t \geq 0$ we have that $\dot{x}(t)=-L v(t)$ with $v(t) \in \mathcal{K} \mathrm{q}(x(t))$ and (see the proof of Theorem 2) as long as $x(t) \notin \overline{\mathcal{D}}$, we also have that for every $t \geq 0$,

$$
\begin{aligned}
\frac{d}{d t} V(x(t)) & =\nabla V(x(t)) \cdot \dot{x}(t) \\
& =-\frac{1}{2} \sum_{i j}\left(x_{j}(t)-x_{i}(t)\right) A_{i j}\left(v_{j}(t)-v_{i}(t)\right) \\
& \leq-\frac{1}{2} \underline{A} \sum_{(i, j) \in E}\left(x_{j}(t)-x_{i}(t)\right)\left(v_{j}(t)-v_{i}(t)\right) \\
& =-\frac{1}{2} \underline{A} \sum_{(i, j) \in E}\left|x_{j}(t)-x_{i}(t)\right|\left|v_{j}(t)-v_{i}(t)\right| \\
& \leq-\frac{1}{2} \underline{A} \max _{(i, j) \in E}\left|x_{j}(t)-x_{i}(t)\right|\left|v_{j}(t)-v_{i}(t)\right| \\
& \leq-\frac{1}{2} \underline{A} \Delta \max _{(i, j) \in E}\left|x_{j}(t)-x_{i}(t)\right| \\
& \leq-\phi \Delta \underline{A},
\end{aligned}
$$

where $\underline{A}=\min _{i, j \in V}\left\{A_{i j}: A_{i j} \neq 0\right\}$ and $\phi=\frac{1}{2} \inf _{t \geq 0}\left\{\max _{(i, j) \in E}\left|x_{j}(t)-x_{i}(t)\right|: x(t) \in \mathbb{R}^{N} \backslash \overline{\mathcal{D}}\right\}$. If $\phi>0$, by integrating over the interval $[0, T]$, we get

$$
V(x(T))-V(x(0)) \leq-\phi \underline{A} \Delta T .
$$

Assume by contradiction that $x(t)$ does not reach $\overline{\mathcal{D}}$ in finite time. Then by letting $T \rightarrow+\infty$ we get that $\lim _{T \rightarrow+\infty} V(x(T))=-\infty$, which contradicts the fact that $V$ is positive definite.

We now prove that if $\phi=0$, then $x_{\text {ave }}(0)=(k+1 / 2) \Delta$ for some $k \in \mathbb{Z}$. If $\phi=0$, then for any $n \in \mathbb{N}$ there exists $t_{n}$ such that $x\left(t_{n}\right) \in \mathbb{R}^{N} \backslash \overline{\mathcal{D}}$ and, for all $(i, j) \in E$, it holds $\left|x_{j}\left(t_{n}\right)-x_{i}\left(t_{n}\right)\right|<\frac{1}{n}$. Let us fix any $k \in V$, and consider a path of length $K$ connecting all the vertices of $G$ and starting from $k$. Let us denote the states of the nodes in this path as $\tilde{x}_{1}, \ldots, \tilde{x}_{K}$, noting that some of the nodes may appear in the list more than once. From the fact that $\phi=0$ we deduce that

$$
\begin{gathered}
\tilde{x}_{1}\left(t_{n}\right)-\frac{1}{n}<\tilde{x}_{2}\left(t_{n}\right)<\tilde{x}_{1}\left(t_{n}\right)+\frac{1}{n} \\
\tilde{x}_{1}\left(t_{n}\right)-\frac{2}{n}<\tilde{x}_{2}\left(t_{n}\right)-\frac{1}{n}<\tilde{x}_{3}\left(t_{n}\right)<\tilde{x}_{2}\left(t_{n}\right)+\frac{1}{n}<\tilde{x}_{1}\left(t_{n}\right)+\frac{2}{n}, \\
\text { etc. } \\
\tilde{x}_{1}\left(t_{n}\right)-\frac{K}{n}<\tilde{x}_{K}\left(t_{n}\right)<\tilde{x}_{1}\left(t_{n}\right)+\frac{K}{n} .
\end{gathered}
$$


By summing $N$ of these inequalities corresponding to the $N$ different nodes we get, since by definition $\tilde{x}_{1}=x_{k}$, that

$$
x_{k}\left(t_{n}\right)-\frac{1}{n} \sum_{i=1}^{K} i<\frac{1}{N} \sum_{i=1}^{N} x_{i}\left(t_{n}\right)<x_{k}\left(t_{n}\right)+\frac{1}{n} \sum_{i=1}^{K} i .
$$

Since $\frac{1}{N} \sum_{i=1}^{N} x_{i}\left(t_{n}\right)=\frac{1}{N} \sum_{i=1}^{N} x_{i}(0)$ we obtain

$$
\left|\frac{1}{N} \sum_{i=1}^{N} x_{i}(0)-x_{k}\left(t_{n}\right)\right|<\frac{K(K+1)}{2 n}
$$

and then $x_{k}\left(t_{n}\right) \rightarrow \frac{1}{N} \sum_{i=1}^{N} x_{i}(0)$ as $n \rightarrow+\infty$. By the arbitrariness of $k$, we get $x\left(t_{n}\right) \rightarrow$ $x_{\text {ave }}(0) \mathbf{1}$ as $n \rightarrow+\infty$. We recall that $x\left(t_{n}\right) \in \mathbb{R}^{N} \backslash \overline{\mathcal{D}}$ for every $n$, then $x_{\text {ave }}(0) \mathbf{1} \in \overline{\mathbb{R}^{N} \backslash \overline{\mathcal{D}}}$. Finally since $\overline{\mathbb{R}^{N} \backslash \overline{\mathcal{D}}} \cap \operatorname{span} \mathbf{1}=\left\{\left(k+\frac{1}{2}\right) \Delta: k \in \mathbb{Z}\right\}$ we get that $x_{\text {ave }}(0)=\left(k+\frac{1}{2}\right) \Delta$ for some $k \in \mathbb{Z}$.

Remark 2 (Comparison with discrete-time consensus) The results of "practical consensus" in Theorem 1 and 2 can be related with results about discrete-time consensus systems, as those in [13] and [16]. In [13], the authors consider $x(t+1)=x(t)-\frac{1}{d_{\max }+\varepsilon} L \mathrm{q}(x(t))$, where $d_{\max }$ is the largest in-degree in $G$, and $\varepsilon>0$. Clearly $\mathcal{D}$ is the set of equilibria for this system, but its limit behavior shows limit cycles which are not contained in the closure of $\mathcal{D}$. However, for some example topologies (rings and complete graphs) the system can be proved to approach average consensus up to the quantizer precision. The paper [16] considers a discrete-state dynamics in which the agents communicate in randomly chosen pairs, and proves convergence to a set of the form $\left\{x \in \mathbb{Z}^{N}: x_{i} \in\{L, L+1\}, L \in \mathbb{Z}\right\}$.

\section{Chattering-free quantized dynamics}

The analysis in Remark 1 has pointed out the existence of sliding modes in the system $\dot{x}=-L \mathrm{q}(x)$, a phenomenon which is not acceptable in practical implementation. In this section we discuss a different quantization scheme to overcome this difficulty and we analyze the resulting system.

\subsection{Hysteretic quantizer: hybrid model}

The different quantization scheme is based on a quantizer with hysteresis which we introduce below. Let us consider the multi-valued map qh defined as (Figure 2)

$$
\mathrm{q}_{\mathrm{h}}(r)=\left\{\begin{array}{lr}
j \Delta & -\frac{\Delta}{2}+j \Delta \leq r<\frac{\Delta}{2}+j \Delta \\
j \Delta+\frac{\Delta}{2} & j \Delta \leq r<\Delta+j \Delta, \quad j \in \mathbb{Z}
\end{array}\right.
$$

for the scalar $r$, with the understanding that if $x \in \mathbb{R}^{n}, \mathrm{q}_{\mathrm{h}}(x)=\left(\mathrm{q}_{\mathrm{h}}\left(x_{1}\right), \ldots, \mathrm{q}_{\mathrm{h}}\left(x_{n}\right)\right)^{*}$ The evolution of $\mathrm{q}_{\mathrm{h}}(x(t))$ as a function of $x(t)$ can be described as follows. At time $t=0$, $\mathrm{q}_{\mathrm{h}}(x(0))=\mathrm{q}(x(0))$. Let, for the sake of notational simplicity, $\mathrm{q}_{\mathrm{h}}:=\mathrm{q}_{\mathrm{h}}(x(t))$, and $\mathrm{q}_{\mathrm{h}}^{+}:=$ $\lim _{s \rightarrow t^{+}} \mathrm{q}_{\mathrm{h}}(x(s)), x:=x(t)$. If $\mathrm{q}_{\mathrm{h}} \in(\Delta \mathbb{Z} \cup(\Delta \mathbb{Z}+\Delta / 2))^{N}$, then

$$
\mathrm{q}_{\mathrm{h}}^{+}=\left\{\begin{array}{cc}
\mathrm{q}_{\mathrm{h}}+\frac{\Delta}{2} & \text { if } x \geq \mathrm{q}_{\mathrm{h}}+\frac{\Delta}{2} \\
\mathrm{q}_{\mathrm{h}}-\frac{\Delta}{2} & \text { if } x \leq \mathrm{q}_{\mathrm{h}}-\frac{\Delta}{2} \\
\mathrm{q}_{\mathrm{h}} & \text { if } \mathrm{q}_{\mathrm{h}}-\frac{\Delta}{2}<x<\mathrm{q}_{\mathrm{h}}+\frac{\Delta}{2} .
\end{array}\right.
$$




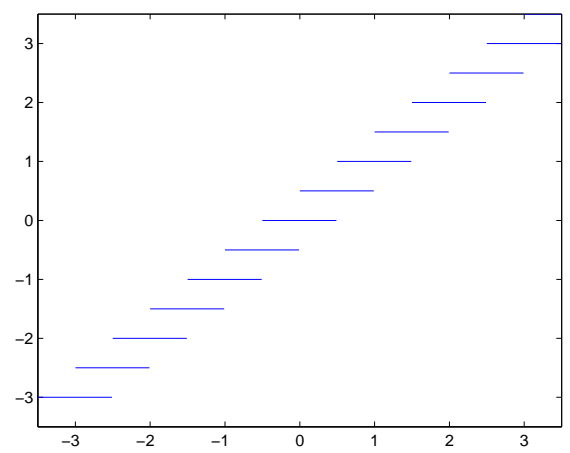

Figure 2: The uniform quantizer with hysteresis $\mathrm{q}_{\mathrm{h}}(z)$, when $\Delta=1$.

The rule (11) is illustrated in Figure 3.

Suppose that in the dynamics (4), each agent quantizes the information using $\mathrm{q}_{\mathrm{h}}$ rather than q. This leads to a system which can be better described and analyzed using the formalism of hybrid systems. To this end, we adopt the notations in [14]. Let $q \in(\Delta \mathbb{Z} \cup$ $(\Delta \mathbb{Z}+\Delta / 2))^{N}$ be the discrete state, $x \in \mathbb{R}^{N}$ the continuous state and $X=\mathbb{R}^{N} \times(\Delta \mathbb{Z} \cup$ $(\Delta \mathbb{Z}+\Delta / 2))^{N}$ the state space where the system evolves. The continuous dynamics of the system are described by

$$
\begin{aligned}
\dot{x} & =-L q \\
\dot{q} & =0
\end{aligned}
$$

which are valid as far as the state $(x, q)$ belongs to the subset of the state space:

$$
C=\left\{(x, q) \in X: \forall i \in V,-\frac{\Delta}{2}+q_{i}<x_{i}<\frac{\Delta}{2}+q_{i}\right\} .
$$

If on the other hand $(x, q)$ belongs to the set

$$
D=\left\{(x, q) \in X: \exists i \in V, \text { such that } x_{i} \leq-\frac{\Delta}{2}+q_{i} \text { or } x_{i} \geq \frac{\Delta}{2}+q_{i}\right\},
$$

then the following discrete update occurs:

$$
\begin{aligned}
& x^{+}=x \\
& q_{i}^{+}= \begin{cases}q_{i}+\frac{\Delta}{2} & \text { if } x_{i} \geq \frac{\Delta}{2}+q_{i} \\
q_{i}-\frac{\Delta}{2} & \text { if } x_{i} \leq-\frac{\Delta}{2}+q_{i} \\
q_{i} & \text { otherwise. }\end{cases}
\end{aligned}
$$

Observe that $C \cup D=X$ and $C \cap D=\emptyset$. The equations (12) and (13) together with the sets $C, D$ define the hybrid model associated with the multi-agent system in the presence of the quantizers (10). In what follows we let $z=\left(x^{*} q^{*}\right)^{*}$ be the entire state of the hybrid model and $f(z), g(z)$ the maps on the righthand side of (12) and, respectively, (13). Hence, the system is concisely described as

$$
\begin{aligned}
\dot{z} & =f(z) \quad z \in C \\
z^{+} & =g(z) \quad z \in D,
\end{aligned}
$$




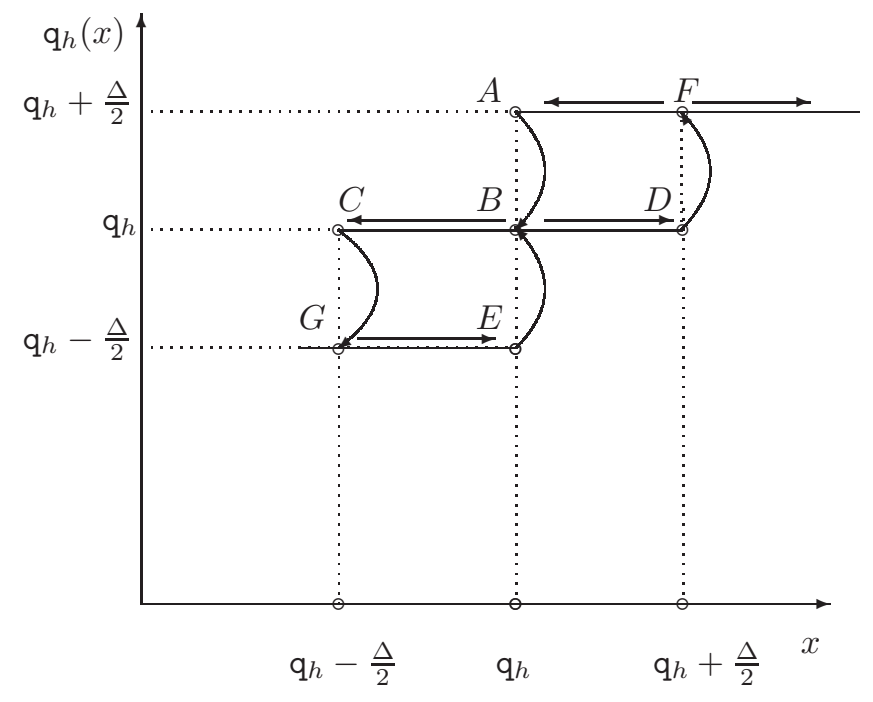

Figure 3: The figure illustrates the evolution of $\mathrm{q}_{h}(x)$ as a function of $x$ as described in (11). Suppose initially that $\mathrm{q}_{\mathrm{h}}-\frac{\Delta}{2}<x<\mathrm{q}_{\mathrm{h}}+\frac{\Delta}{2}$ and that $\mathrm{q}_{\mathrm{h}}(x)=\mathrm{q}_{\mathrm{h}}$. As $x$ evolves, the point $\left(x, \mathrm{q}_{\mathrm{h}}(x)\right)$ lies on the segment $\overline{C D}$, and it may hit the point $C$ or the point $D$, thus triggering a discrete transition. If the former occurs, the discrete value takes a new value, namely $\mathrm{q}_{\mathrm{h}}^{+}=\mathrm{q}_{\mathrm{h}}-\frac{\Delta}{2}$, the point $\left(x, \mathrm{q}_{\mathrm{h}}(x)\right)$ jumps to $G$ and can then move towards $E$ or in the opposite direction. On the other hand, if $\left(x, \mathrm{q}_{\mathrm{h}}(x)\right)$ hits $D$, then the quantization level takes the new value $\mathrm{q}_{\mathrm{h}}+\frac{\Delta}{2}$, i.e. $\mathrm{q}_{\mathrm{h}}^{+}=\mathrm{q}_{\mathrm{h}}+\frac{\Delta}{2}$. In the graph, this corresponds to the transition from point $D$ to point $F$. The state $x$ can then further increase or decrease. 
with an initial condition belonging to

$$
X_{0}=\left\{(x, q) \in X: q_{i}-\frac{\Delta}{2} \leq x_{i}<q_{i}+\frac{\Delta}{2}, \forall i \in V\right\} .
$$

Note that initial conditions of the form $\left(x_{0}, \mathrm{q}\left(x_{0}\right)\right)$, with $x_{0} \in \mathbb{R}^{N}$, belong to this set.

We recall from [14] the notion of hybrid time domain and solution for a hybrid system. A hybrid time domain is a subset of $\mathbb{R}_{\geq 0} \times \mathbb{N}$ which is the union of infinitely many intervals of the form $\left[t_{j}, t_{j+1}\right] \times\{j\}$, where $0=t_{0} \leq t_{1} \leq t_{2} \leq \ldots$, or of finitely many such intervals with the last one possibly of the form $\left[t_{j}, t_{j+1}\right] \times\{j\},\left[t_{j}, t_{j+1}\right) \times\{j\}$, or $\left[t_{j},+\infty\right) \times\{j\}$. Let $z(t, j)$ be a function defined on a hybrid time domain $\operatorname{dom} z$ such that for each fixed $j, t \mapsto z(t, j)$ is a locally absolutely continuous function on the interval $I_{j}=\{t:(t, j) \in \operatorname{dom} z\}$. The function $z(t, j)$ is a solution to the hybrid system (14) if $z(0,0) \in C \cup D$ and the following conditions are satisfied:

- For each $j$ such that $I_{j}$ has non-empty interior,

$$
\begin{array}{ll}
\dot{z}(t, j)=f(z(t, j)) & \text { for a.e. } t \in I_{j} \\
z(t, j) \in C & \text { for all } t \in\left[\min I_{j}, \sup I_{j}\right)
\end{array}
$$

- For each $(t, j) \in \operatorname{dom} z$ such that $(t, j+1) \in \operatorname{dom} z$,

$$
\begin{aligned}
z(t, j+1) & =g(z(t, j)) \\
z(t, j) & \in D .
\end{aligned}
$$

The solution $z$ is nontrivial if dom $z$ contains at least another point different from $(0,0)$ and is complete if $\operatorname{dom} z$ is unbounded.

\subsection{Hybrid model analysis}

In this subsection we detail the analysis of system (14). After proving basic properties about existence, uniqueness and completeness of solutions, we verify that chattering can not occur. We also compute the equilibria of the system, and present a convergence result.

Lemma 5 (Basic properties of solutions) For each $z(0,0) \in X_{0}$, there exists a nontrivial solution $z(t, j)$ to (14), and such solution is forward unique and complete. Moreover, every interval $I_{j}=\{t:(t, j) \in \operatorname{dom} z\}$, possibly with the exception of $I_{0}$, has a non-void interior.

Proof: There are two cases: $z(0,0) \in D$ or $z(0,0) \in C$. If $z(0,0) \in D$, then $z(0,1) \in C$. Indeed, let $i \in V$ be any index such that $x_{i}(0,0)=\frac{\Delta}{2}+q_{i}(0,0)$. Then $x_{i}(0,1)=x_{i}(0,0)$ and $q_{i}(0,1)=\frac{\Delta}{2}+q_{i}(0,0)$, from which $x_{i}(0,1)=q_{i}(0,1)$. Similarly, for any index $k$ for which $x_{k}(0,0)=-\frac{\Delta}{2}+q_{k}(0,0)$, we have $x_{k}(0,1)=x_{k}(0,0)$ and $q_{k}(0,1)=-\frac{\Delta}{2}+q_{k}(0,0)$. For all the remaining indices $\ell, q_{\ell}(0,0)-\frac{\Delta}{2}<x_{\ell}(0,0)<q_{\ell}(0,0)+\frac{\Delta}{2}$ and then $q_{\ell}(0,1)=q_{\ell}(0,0)$ and $x_{\ell}(0,1)=x_{\ell}(0,0)$. Then $z(0,1) \in C$ and the discrete transition stops in one step. Since the righthand side of (12) is constant, the solution $z(t, j)$ starting either from $z(0,1)$ (if $z(0,0) \in D$ ) or from $z(0,0)$ (if $z(0,0) \in C$ ) exists for $t$ sufficiently close to 0 and is unique. As a matter of fact, one can compute explicitly the solution $z(t, j)$ on the interval $I_{j}$, with $j=0$ (if $z(0,0) \in C$ ) or $j=1$ (if $z(0,0) \in D$ ). Define the auxiliary Cauchy problem $\dot{\zeta}(t)=-L q(0, j), \zeta(0)=x(0, j)$, where by a slight abuse of notation we are denoting the time variable in the auxiliary system by the same symbol $t$ which appears in the hybrid 
time $(t, j)$. The problem admits the unique solution $\zeta(t)$ which is defined for all $t \geq 0$. Let $\bar{t}$ be the minimal time greater than zero for which there exists an index $k \in V$ such that $\zeta_{k}(\bar{t})=-\frac{\Delta}{2}+q_{k}(0, j)$ or $\zeta_{k}(\bar{t})=\frac{\Delta}{2}+q_{k}(0, j)$. Set $I_{j}=[0, \bar{t}]$ if a finite $\bar{t}$ exists, otherwise set $I_{j}=[0,+\infty)$. Finally let $z(t, j)=\left(\zeta(t)^{*} q(0, j)^{*}\right)^{*}$ for all $t \in I_{j}$. Then, by construction, $z(t, j)$ satisfies the condition (12). If $\sup I_{j}=+\infty$, we have concluded the argument. If instead $\sup I_{j}<+\infty, z(t, j)$ satisfies the differential equation $\dot{z}(t, j)=f(z(t, j))=-L q(0, j)$ for all $t \in I_{j}$ except at $t=\sup I_{j}$; but $z\left(\sup I_{j}, j\right) \in D$ and at time $t=\sup I_{j}$ a discrete transition occurs. After this transition, the solution can be uniquely extended starting from $z\left(\sup I_{j}, j+1\right) \in C$, repeating the argument which have been just discussed. Then, $z(t, j)$ is forward unique because it results from the concatenation of unique solutions to (12) and (13).

Let us show that the solution is complete. By contradiction, domz is bounded, that is domz is the union of finitely many intervals of the form $\left[t_{j}, t_{j+1}\right] \times\{j\}$, with the last interval either of the form $\left[t_{j}, t_{j+1}\right] \times\{j\}$ or $\left[t_{j}, t_{j+1}\right) \times\{j\}$ and $t_{j+1}<+\infty$. This yields a contradiction, because in view of the form of the last interval, no more discrete transitions take place and by (12) the continuous evolution exists for all $t$.

Finally, the interval $I_{j}=\{t:(t, j) \in \operatorname{dom} z\}$, with $j$ such that $z(0, j) \in C$, has a non-void interior because the solution to $(14)$ from $z(0, j)$ flows continuously with bounded velocity. If $\sup I_{j}=+\infty$, then $\left(I_{j}, j\right)$ is the last interval of dom $z$ and the proof ends. Otherwise, if $\sup I_{j}<+\infty$, then by definition $z\left(\sup I_{j}, j\right) \in D$ and there is an index $i \in V$ such that either $x_{i}\left(\sup I_{j}, j\right)=\frac{\Delta}{2}+q_{i}\left(\sup I_{j}, j\right)$ or $x_{i}\left(\sup I_{j}, j\right)=-\frac{\Delta}{2}+q_{i}\left(\sup I_{j}, j\right)$. Then, as in the first part of the proof, one proves that $z\left(\sup I_{j}, j+1\right) \in C$ and again $I_{j+1}$ has a non-empty interior because the system flows continuously with bounded velocity. The thesis descends by induction.

The following lemma proves boundedness of solutions, a property which is useful in order to prove that chattering does not occur.

Lemma 6 (Bounded solutions) For every initial condition $z(0,0) \in X_{0}$, the solution $z(t, j)$ satisfies

$$
\begin{aligned}
& -\frac{\Delta}{2}+\min \left\{q_{1}(0,0), \ldots, q_{N}(0,0)\right\} \leq q_{i}(t, j) \leq \max \left\{q_{1}(0,0), \ldots, q_{N}(0,0)\right\}+\frac{\Delta}{2} \\
& -\Delta+\min \left\{q_{1}(0,0), \ldots, q_{N}(0,0)\right\} \leq x_{i}(t, j) \leq \max \left\{q_{1}(0,0), \ldots, q_{N}(0,0)\right\}+\Delta,
\end{aligned}
$$

for all $i \in V$ and for all $(t, j) \in \operatorname{dom} z$.

Proof: Consider the function $U(z)=\max \left\{q_{1}, \ldots, q_{N}\right\}$ and compute it along the solution to (14). During continuous evolutions, i.e. if $z(t, j) \in C, U(z(t))$ remains constant since, by the second equation in (12), no component of $q(t, j)$ changes its value. If $z(t, j) \in D$ and $(t, j) \neq(0,0)$, then $U(z(t, j+1)) \leq U(z(t, j))$. In fact, suppose this is not true. Then, since $q(t, j) \in(\Delta \mathbb{Z} \cup(\Delta \mathbb{Z}+\Delta / 2))^{N}$, and at the transition each component $q_{i}$ either remains the same or changes its value by $\pm \Delta / 2$, i.e. $q_{i}(t, j+1)=q_{i}(t, j)$ or $q_{i}(t, j+1)=q_{i}(t, j) \pm \Delta / 2$, the only possibility for having $U(z(t, j+1))>U(z(t, j))$ is that one of the agents, say $k$, for which its discrete state $q_{k}(t, j)$ equals $\max \left\{q_{1}(t, j), \ldots, q_{N}(t, j)\right\}$, increases its value of $+\Delta / 2$. But for this to occur, it must be true that during the continuous evolution which preceded the transition, the continuous state $x_{k}(t, j)$ has increased its value until it reached the threshold $\frac{\Delta}{2}+q_{k}(t, j)=x_{k}(t, j)$. This is a contradiction because, since $q_{k}(t, j)=\max \left\{q_{1}(t, j), \ldots, q_{N}(t, j)\right\}$, in the equation $\dot{x}_{k}(t, j)=\sum_{j=1}^{N} A_{i j}\left(q_{j}(t, j)-q_{k}(t, j)\right)$ the righthand side is non-positive. Hence, we conclude that $U(z(t, j)) \leq U(z(0, k))$ for all $(t, j) \in \operatorname{dom} z$ such that $t \geq 0$ and $j \geq k$, where $k \in\{0,1\}$ is the smallest integer 
such that $z(0, k) \in C$. In case $k=0$, then $U(z(t, j)) \leq U(z(0,0))$. In case $k=1$, then $U(z(0,1)) \leq U(z(0,0))+\Delta / 2$, and $U(z(t, j)) \leq U(z(0,1)) \leq U(z(0,0))+\Delta / 2$. Similarly one can prove that $W(z)=\min \left\{q_{1}, \ldots, q_{N}\right\}$ satisfies $W(z(t, j)) \geq W(z(0,0))-\Delta / 2$.

The conclusion on the continuous state follows easily since $z(0,0) \in X_{0}$ implies that for each $(t, j) \in \operatorname{dom} z$, for each $i \in V, q_{i}(t, j)-\frac{\Delta}{2} \leq x_{i}(t, j) \leq q_{i}(t, j)+\frac{\Delta}{2}$.

The last part of the thesis of Lemma 5 implies that the set $\left\{t \in \mathbb{R}_{\geq 0} \mid z(t, j) \in D\right\}$ has a zero measure. Moreover, a finer analysis can be performed. Let us consider the switching times of a solution $z(t, j)$ to the hybrid system originating from $z(0,0) \in X_{0}$, i.e. the times $t_{j}$ such that $\left(t_{j}, j\right) \in \operatorname{dom} z \operatorname{implies}\left(t_{j}, j+1\right) \in \operatorname{dom} z$. The set of switching times is locally finite if, for any compact subset of $\mathbb{R}_{\geq 0}$, there is only a finite number of switching times which belong to that compact subset.

Proposition 5 (No chattering) For every initial condition $z(0,0) \in X_{0}$, the set of switching times of the solution $z(t, j)$ is locally finite.

Proof: First of all we remark that for each agent $j$, each time a switching occurs, there must elapse an interval of time of length at least $\frac{\Delta / 2}{\|L\|_{\infty}\left(\|q(0,0)\|_{\infty}+\Delta / 2\right)}$ before the agent $i$ switches again: that is, for each agent the inter-switching intervals have lengths which are bounded from below. Indeed, the agent's state $x_{i}(t, j)$ evolves with speed not larger than $\|L\|_{\infty}\left(\|q(0,0)\|_{\infty}+\Delta / 2\right)$ (see Lemma 6) and, after each switching, it has to cover a distance of length at least $\Delta / 2$ before it fulfills a switching condition again.

Let us now suppose that there exists a solution $z(t, j)$ and a sequence of switching times $t_{j}$, with $\left(t_{j}, j\right) \in \operatorname{dom} z$, such that $j \rightarrow \infty$ and

$$
\lim _{j \rightarrow+\infty} \sum_{k=0}^{j}\left(t_{k+1}-t_{k}\right)=T<+\infty .
$$

Because the number of switches is infinite and the number of agents is finite, then there is at least an index $i \in V$ for which $q_{i}$ switches infinitely many times. We can then extract the sequence $t_{i_{k}}$ of times at which $q_{i}$ changes its value. Clearly,

$$
\lim _{j \rightarrow+\infty} \sum_{k=0}^{j}\left(t_{i_{k+1}}-t_{i_{k}}\right)<+\infty .
$$

On the other hand, thanks to the previous argument, one has that

$$
t_{i_{k+1}}-t_{i_{k}} \geq \frac{\Delta / 2}{\|L\|_{\infty}\left(\|q(0,0)\|_{\infty}+\Delta / 2\right)},
$$

and then

$$
\lim _{j \rightarrow+\infty} \sum_{k=0}^{j}\left(t_{i_{k+1}}-t_{i_{k}}\right)=+\infty
$$

i.e. a contradiction.

Remark 3 (On the "continuous component" of the hybrid time domain) Combining Lemma 5 and Proposition 5 it is possible to conclude that the continuous component of the hybrid time domain is unbounded. Namely domz is either the union of infinitely many intervals of the form $\left[t_{j}, t_{j+1}\right] \times\{j\}$, with $0=t_{0} \leq t_{1} \leq \ldots$, or the union of finitely many of such intervals, the last one being $\left[t_{j},+\infty\right) \cup\{j\}$. Moreover, in the former case, necessarily $\cup_{j \in \mathbb{N}}\left[t_{j}, t_{j+1}\right]=\mathbb{R}_{\geq 0}$ by Proposition 5 . 
Remark 4 (Data rate) In the case of quantizers with hysteresis, thanks to Lemma 6 and Proposition 5, one can find an upper bound on the the data rate at which each agent transmits information to its neighbors. In fact, the proof of Proposition 5 tells us that the information regarding the state is transmitted at most every $T:=\frac{\Delta / 2}{\|L\|_{\infty}\left(\|q(0,0)\|_{\infty}+\Delta / 2\right)}$ units of time. The information consists of packets of bits which encode the quantized state of the agents. In view of Lemma 6 , the number of quantization levels employed by each agent is $4 \frac{\|q(0,0)\|_{\infty}+\Delta / 2}{\Delta / 2}+1$. Hence, to encode these quantization levels, $B:=\left\lceil\log _{2}\left(8 \frac{\|q(0,0)\|_{\infty}}{\Delta}+5\right)\right\rceil$ bits are needed. We conclude that each agent transmits information at a data rate which is not larger than

$$
\frac{B}{T}=\left\lceil\log _{2}\left(8 \frac{\|q(0,0)\|_{\infty}}{\Delta}+5\right)\right\rceil\left(2 \frac{\|q(0,0)\|_{\infty}}{\Delta}+1\right)\|L\|_{\infty} .
$$

We can also prove that the solution to (14) preserves the average of the continuous states.

Lemma 7 (Average preservation) For each $z(0,0) \in X_{0}$, the solution $z(t, j)$ to (14) is such that $N^{-1} \mathbf{1}^{*} x(t, j)=N^{-1} \mathbf{1}^{*} x(0,0)$, for all $(t, j) \in \operatorname{dom} z$.

Proof: $\quad$ By (13), for all $(t, j) \in \operatorname{dom} z$ such that $(t, j+1) \in \operatorname{dom} z, x(t, j)=x(t, j+1)$, i.e. during discrete transitions the value of $x$ does not change and therefore the average is trivially preserved. For $z(t, j) \in C$, the continuous state $x(t, j)$ satisfies $N^{-1} \mathbf{1}^{*} \dot{x}(t, j)=$ $-N^{-1} \mathbf{1}^{*} L q(t, j)$ for all $t \in I_{j}$. Since the graph is weight-balanced, then $\mathbf{1}^{*} L=\mathbf{0}^{*}$, and therefore the average is preserved also during continuous flow. This leads to the thesis.

Consider now the set of equilibria of the system, i.e. the set of states such that all the evolutions originating from those remain in the same state. This set is characterized in the following statement.

Lemma 8 (Equilibria) The set of equilibria for the system (14) is $\mathcal{E}=\mathcal{E}_{1} \cup \mathcal{E}_{2}$, with

$$
\begin{aligned}
& \mathcal{E}_{1}=\left\{(x, q) \in X: \exists k \in \mathbb{Z} \text { s.t. } q_{i}=k \Delta, \text { and }-\frac{\Delta}{2}+q_{i}<x_{i}<\frac{\Delta}{2}+q_{i}, \forall i \in V\right\} \\
& \mathcal{E}_{2}=\left\{(x, q) \in X: \exists k \in \mathbb{Z} \text { s.t. } q_{i}=k \Delta+\frac{\Delta}{2}, \text { and }-\frac{\Delta}{2}+q_{i}<x_{i}<\frac{\Delta}{2}+q_{i}, \forall i \in V\right\} .
\end{aligned}
$$

Proof: Suppose that the initial condition $z(0,0)$ belongs to $\mathcal{E}_{1}$. Then, since $q_{i}(0,0)=$ $k \Delta$ and $-\frac{\Delta}{2}+q_{i}(0,0)<x_{i}(0,0)<\frac{\Delta}{2}+q_{i}(0,0)$ for all $i \in V, z(0,0) \in C$, no discrete transition is triggered, and moreover $-L q(0,0)=\mathbf{0}$, i.e. during the continuous flow the state remains unchanged. The same can be argued if the initial condition $z(0,0)$ belongs to $\mathcal{E}_{2}$.

Suppose now that $z(0,0) \in X$ is an equilibrium point, i.e. $z(t, j)=z(0,0)$ for all $(t, j) \in$ $\operatorname{dom} z$. Then $z(0,0) \notin D$, because otherwise a discrete update via (13) would occur leading to $q(0,1) \neq q(0,0)$, a contradiction. Since $X=C \cup D$, then $z(0,0) \in C$. In order to have $x(t, 0)=x(0,0)$ for all $t \in I_{0}$, it must necessarily be true that $-L q(0,0)=\mathbf{0}$, i.e. $q(0,0) \in$ $\operatorname{span} 1 \cap(\Delta \mathbb{Z} \cup(\Delta \mathbb{Z}+\Delta / 2))^{N}$ or, equivalently, that for each $i \in V$, either $q_{i}(0,0)=k \Delta+\frac{\Delta}{2}$ or $q_{i}(0,0)=k \Delta$ for some $k \in \mathbb{Z}$. Since $z(0,0) \in C$, then necessarily $-\frac{\Delta}{2}+q_{i}(0,0)<x_{i}(0,0)<$ $\frac{\Delta}{2}+q_{i}(0,0)$, for all $i \in V$. We conclude that $z(0,0) \in \mathcal{E}_{1} \cup \mathcal{E}_{2}$.

Differently from the case of quantizers with no hysteresis, the set of equilibria $\mathcal{E}$ may not be globally attractive for the solutions to (14) as the following example shows. 
Example 2 (Finite-time limit cycle) Consider the system (12)-(13) with $N=2$ and where $\dot{x}=-L q$ is

$$
\begin{aligned}
& \dot{x}_{1}=q_{2}-q_{1} \\
& \dot{x}_{2}=q_{1}-q_{2} .
\end{aligned}
$$

Let $q_{1} \in \Delta \mathbb{Z}$ be fixed and consider the initial condition

$$
z_{1}(0,0)=\left(q_{1}-\frac{\Delta}{4}, q_{1}\right), z_{2}(0,0)=\left(q_{1}+\frac{3 \Delta}{4}, q_{1}+\Delta\right) .
$$

The continuous dynamics is given by

$$
\begin{aligned}
& \dot{x}_{1}=\Delta \\
& \dot{x}_{2}=-\Delta
\end{aligned}
$$

so that

$$
\begin{aligned}
& x_{1}(t)=q_{1}-\frac{\Delta}{4}+\Delta t \\
& x_{2}(t)=q_{1}+\frac{3 \Delta}{4}-\Delta t
\end{aligned}
$$

on the interval $\left[0, \frac{1}{4}\right] \times\{0\}$. Indeed, at time $t=\frac{1}{4}$ we have $x_{1}\left(\frac{1}{4}\right)=q_{1}, x_{2}\left(\frac{1}{4}\right)=q_{1}+\frac{\Delta}{2}$, so that a discrete transition is triggered. It holds

$$
z_{1}\left(\frac{1}{4}, 1\right)=\left(q_{1}, q_{1}\right), \quad z_{2}\left(\frac{1}{4}, 1\right)=\left(q_{1}+\frac{\Delta}{2}, q_{1}+\frac{\Delta}{2}\right) .
$$

The continuous dynamics is then given by

$$
\begin{aligned}
& \dot{x}_{1}=\frac{\Delta}{2} \\
& \dot{x}_{2}=-\frac{\Delta}{2}
\end{aligned}
$$

so that

$$
\begin{aligned}
& x_{1}(t)=q_{1}+\frac{\Delta}{2}\left(t-\frac{1}{4}\right) \\
& x_{2}(t)=q_{1}+\frac{\Delta}{2}-\frac{\Delta}{2}\left(t-\frac{1}{4}\right)
\end{aligned}
$$

on the interval $\left[\frac{1}{4}, \frac{5}{4}\right] \times\{1\}$. At time $t=\frac{5}{4}$ we have $x_{1}\left(\frac{5}{4}\right)=q_{1}+\frac{\Delta}{2}, x_{2}\left(\frac{5}{4}\right)=q_{1}$, so that a new discrete transition is triggered. We have:

$$
z_{1}\left(\frac{5}{4}, 2\right)=\left(q_{1}+\frac{\Delta}{2}, q_{1}+\frac{\Delta}{2}\right), \quad z_{2}\left(\frac{5}{4}, 2\right)=\left(q_{1}, q_{1}\right) .
$$

In the next interval the continuous dynamics is given by

$$
\begin{aligned}
& \dot{x}_{1}=-\frac{\Delta}{2} \\
& \dot{x}_{2}=\frac{\Delta}{2}
\end{aligned}
$$

so that

$$
\begin{aligned}
& x_{1}(t)=q_{1}+\frac{\Delta}{2}-\frac{\Delta}{2}\left(t-\frac{5}{4}\right) \\
& x_{2}(t)=q_{1}+\frac{\Delta}{2}\left(t-\frac{5}{4}\right) .
\end{aligned}
$$

At time $t=\frac{9}{4}$ we have $x_{1}\left(\frac{9}{4}\right)=q_{1}=x_{1}\left(\frac{1}{4}\right)$ and $x_{2}\left(\frac{9}{4}\right)=q_{1}+\frac{\Delta}{2}=x_{2}\left(\frac{1}{4}\right)$, so that a new discrete transition is triggered. Hence, $z\left(\frac{9}{4}, 3\right)=z\left(\frac{1}{4}, 1\right)$ and the evolution has entered a cycle.

The conclusion is that there exist trajectories of system (12)-(13) which converge to periodic trajectories in finite time, and in particular do not converge to the set of equilibria. In this bidimensional example, the exhibited periodic trajectory lies in the closure of the set of equilibria. 
It should be noted that finite-time convergence of trajectories implies that solutions to (14) are not backward unique, whereas forward uniqueness has been proved in Lemma 5 .

In view of the existence of limit cycles, we can prove a convergence result analogous to Corollary 1.

Theorem 3 (Finite-time convergence) Consider the system (12)-(13). Then, for any $\varepsilon \in(0,1)$, there exists $T(\varepsilon)>0$ such that the solution $z(t, j)$ to (12)-(13) satisfies

$$
\left\|x(t, j)-\frac{\mathbf{1 1}^{*}}{N} x(0,0)\right\| \leq \frac{1}{1-\varepsilon} \frac{\|L\|}{\lambda_{2}(\operatorname{Sym}(L))} \frac{\Delta}{2} \sqrt{N}
$$

for all $(t, j) \in \operatorname{dom} z$ such that $t \geq T(\varepsilon)$.

Proof: The proof follows the lines of those for Theorem 1 and Corollary 1. Define $y(t, j)=\Omega x(t, j)=\left(I-\frac{\mathbf{1 1} *}{N}\right) x(t, j)$ and observe that for $z(t, j) \in C, y(t, j)$ satisfies

$$
\dot{y}(t, j)=\left(I-\frac{11^{*}}{N}\right) \dot{x}(t, j)=-\left(I-\frac{11^{*}}{N}\right) L q(t, j)=-L q(t, j),
$$

while at each switching time $t_{i}, y\left(t_{i}, i+1\right)=y\left(t_{i}, i\right)$. Given $V(y)=\frac{y^{*} y}{2}$, we investigate $\frac{d V(y(t, j))}{d t}=\nabla V(y(t, j)) \dot{y}(t, j)$ when $z(t, j) \in C$. By Lemma 1, we obtain:

$$
\begin{aligned}
\nabla V(y)(-L q) & =\nabla V(y)(-L(x+q-x)) \\
& =-\nabla V(y) L x-\nabla V(y) L(q-x) \\
& =-\nabla V(y) L y-\nabla V(y) L(q-x) \\
& \leq-\lambda_{2}(\operatorname{Sym}(L))\|y\|^{2}+\|y\|\|L\| \frac{\Delta}{2} \sqrt{N} \\
& =-\lambda_{2}(\operatorname{Sym}(L))\|y\|\left(\|y\|-\frac{\|L\|}{\lambda_{2}(\operatorname{Sym}(L))} \frac{\Delta}{2} \sqrt{N}\right),
\end{aligned}
$$

where in the inequality we exploit the fact that $z \in C$ implies $q_{i}-\frac{\Delta}{2} \leq x_{i} \leq q_{i}+\frac{\Delta}{2}$ and hence $\left|x_{i}-q_{i}\right| \leq \frac{\Delta}{2}$ for all $i \in V$. If

$$
\|y\|>\frac{1}{1-\varepsilon} \frac{\|L\|}{\lambda_{2}(\operatorname{Sym}(L))} \frac{\Delta}{2} \sqrt{N}
$$

then

$$
\nabla V(y)(-L q) \leq-\varepsilon \lambda_{2}(\operatorname{Sym}(L))\|y\|^{2}=-2 \varepsilon \lambda_{2}(\operatorname{Sym}(L)) V(y) .
$$

Hence, for all $y(t, j) \in C$ which satisfy (17), it holds

$$
\frac{d V(y(t, j))}{d t} \leq-2 \varepsilon \lambda_{2}(\operatorname{Sym}(L)) V(y(t, j)) .
$$

At each switching time $\left(t_{i}, i\right)$, on the other hand, we have $V\left(y\left(t_{i}, i+1\right)\right)=V\left(y\left(t_{i}, i\right)\right)$. As a consequence, denoted by $t_{0}=0, t_{1}, t_{2}, \ldots, t_{j}$ the switching times which precede $t$, we have

$$
\begin{aligned}
V(y(t, j)) & \leq \mathrm{e}^{-2 \varepsilon \lambda_{2}(\operatorname{Sym}(L))\left(t-t_{j}\right)} V\left(y\left(t_{j}, j\right)\right) \\
& \leq \mathrm{e}^{-2 \varepsilon \lambda_{2}(\operatorname{Sym}(L))\left(t-t_{j}\right)} V\left(y\left(t_{j}, j-1\right)\right) \\
& \leq \mathrm{e}^{-2 \varepsilon \lambda_{2}(\operatorname{Sym}(L))\left(t-t_{j}\right)} \mathrm{e}^{-2 \varepsilon \lambda_{2}(\operatorname{Sym}(L))\left(t_{j}-t_{j-1}\right)} V\left(y\left(t_{j-1}, j-1\right)\right), \\
& \vdots \\
& \leq \mathrm{e}^{-2 \varepsilon \lambda_{2}(\operatorname{Sym}(L))\left(t-t_{j}\right)} \mathrm{e}^{-2 \varepsilon \lambda_{2}(\operatorname{Sym}(L))\left(t_{j}-t_{j-1}\right)} \ldots \mathrm{e}^{-2 \varepsilon \lambda_{2}(\operatorname{Sym}(L))\left(t_{1}-t_{0}\right)} V\left(y\left(t_{0}, 0\right)\right),
\end{aligned}
$$


which implies

$$
V(y(t, j)) \leq \mathrm{e}^{-2 \varepsilon \lambda_{2}\left(\operatorname{Sym}(L)\left(t-t_{0}\right)\right.} V\left(y\left(t_{0}, 0\right)\right)
$$

and therefore

$$
\|y(t, j)\| \leq \mathrm{e}^{-\varepsilon \lambda_{2}(\operatorname{Sym}(L))\left(t-t_{0}\right)}\left\|y\left(t_{0}, 0\right)\right\| .
$$

From the latter we conclude that, if at time $\left(t_{0}, 0\right)$, condition $(17)$ is satisfied, then after at most

$$
T(\varepsilon)=\max \left\{0, \frac{-1}{\varepsilon \lambda_{2}(\operatorname{Sym}(L))} \ln \left(\frac{1}{1-\varepsilon} \frac{\|L\|}{\lambda_{2}(\operatorname{Sym}(L))} \frac{\Delta}{2} \frac{\sqrt{N}}{\left\|y\left(t_{0}, 0\right)\right\|}\right)\right\}
$$

units of time, $\|y(t, j)\|$, with $t \geq T(\varepsilon)$, satisfies

$$
\|y\|<\frac{1}{1-\varepsilon} \frac{\|L\|}{\lambda_{2}(\operatorname{Sym}(L))} \frac{\Delta}{2} \sqrt{N}
$$

and continue to do so from that time on. The thesis then follows, recalling the definition of $y(t, j)$ and that thanks to Lemma $7, \mathbf{1}^{*} x(t, j)=\mathbf{1}^{*} x(0,0)$ for all $(t, j)$.

One might wonder whether it is possible to make a stronger claim of convergence to a set which does not depend on the network topology, for instance to the closure of the set of equilibria. The answer seems to be negative in general, as we show in the next subsection for systems with more than two agents by means of simulation examples: limit cycles are inherent to the hysteretic dynamics. An interesting open question, which we touch upon commenting the simulations, is to relate these limit cycles, and in particular their size, to the graph topology.

Remark 5 (Convergence rate) From the proofs of Corollary 1 and of Theorem 3 we can see that, away from a strip containing the equilibria, the rate of convergence can be bounded by the same quantity for both algorithms. Actually, the rate is proportional to $\lambda_{2}(\operatorname{Sym}(L))$, which is known to be the rate of convergence of the non-quantized linear consensus dynamics. In other words, our results suggest that the use of quantized measurements, with or without hysteresis, does not affect the rate of convergence of the consensus dynamics.

\subsection{Simulations}

In this section, we collect a few simulation examples, focusing on the effects of hysteresis and on the convergence properties of (14). We performed our simulations using a Matlab program which implements the hybrid system (14), and solves systems (5) and (12) by an explicit Euler scheme with time step $\delta t=0.005$. In the set of simulations we show here, we assume that $N=10, A_{i j} \in\{0,1\}$ for every $i, j \in V$, and $\Delta=0.05$, and that the initial condition is $x(0)=[0.91728,0.26898,0.76538,0.18858,0.28738,0.09098,0.57608,0.68328,0.54648,0.42558]^{*}$. Let us first consider the two networks depicted in Figure 4. Figures 5 and 6 show for these networks the computed evolutions of (5) and (14), in terms of the quantized states $\mathrm{q}(x(t))$ and $q(t, j)$. Notice that solutions to (5) suffer from chattering. Indeed, there are subgraphs of the graph in Figure 4(b) which coincide with the graph discussed in Example 1. Hence, the chattering observed in the simulations is due to a sliding mode similar to the one described in the example and to the numerical implementation of the algorithm. Instead, solutions to (14) show no chattering: as expected, hysteresis prevents chattering, and may affect the convergence rate near the equilibria. The latter statement is not in contrast with Remark 5. In both the examples above, the quantized states of system (14) converge to 


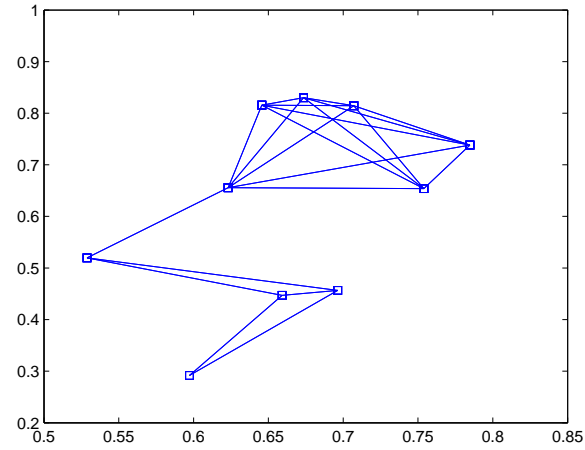

(a)

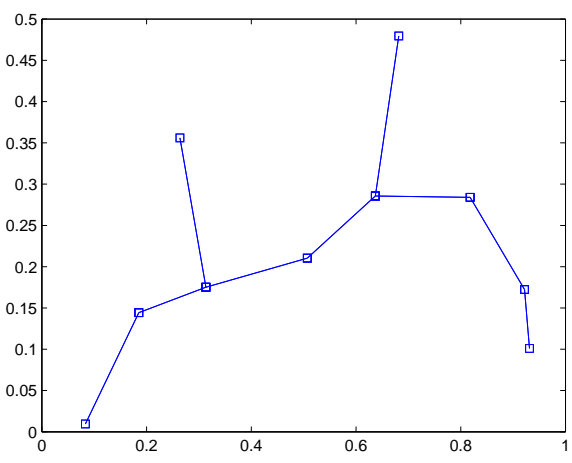

(b)

Figure 4: Two sample graphs, obtained as realizations of a random geometric graph [21] in the unit square $[0,1]^{2}$ with connectivity radius equal to 0.2 .
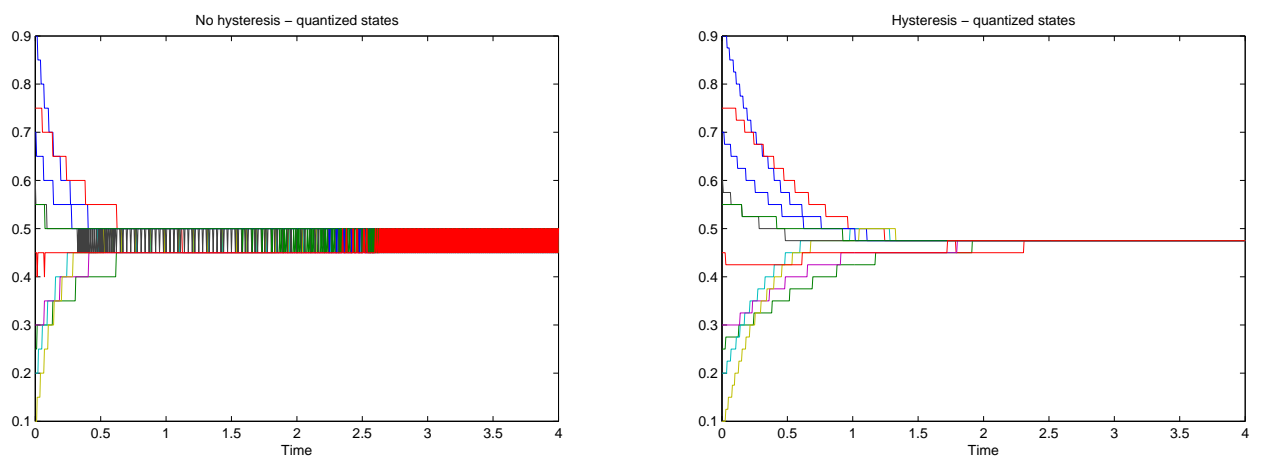

Figure 5: Examples of evolutions of quantized dynamics on the graph in Figure 4(a).

consensus. To provide an example of limit cycles, we consider a directed ring topology, that is a graph such that $\mathcal{N}_{i}=\{i+1\}$ for $i \in\{1, \ldots, N-1\}$ and $\mathcal{N}_{N}=1$.Figure 7 shows that in this case hysteresis induces a limit cycle of amplitude $2 \Delta$ on the quantized states. Furthermore, simulations demonstrate that larger cycles can be obtained on larger rings.

\section{Conclusion}

In this paper we analyzed a consensus problem subject to quantized communication, in terms of the stabilization of a dynamical system by means of a discontinuous feedback. Two quantization rules have been considered: a uniform static quantizer, and a hysteretic quantizer, which we designed with the goal of avoiding chattering phenomena. The convergence properties of both the resulting systems have been studied, and illustrated through simulations. Future work should include the analysis of other feedback consensus dynamics with quantization effects, and the application of these techniques to the problem of guaranteeing stable flocking of autonomous vehicles via quantized feedback control. 

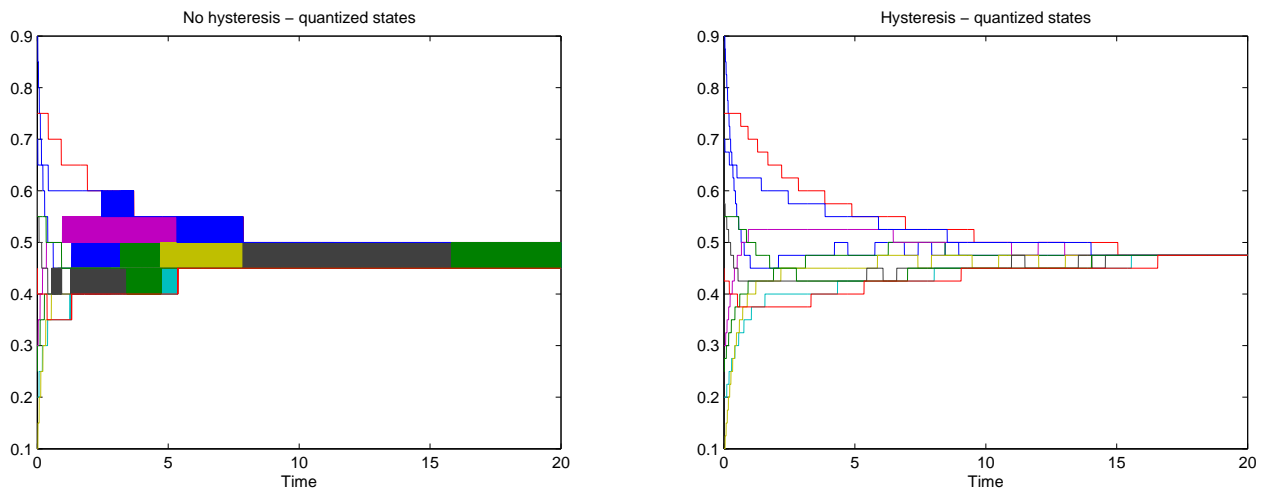

Figure 6: Examples of evolutions of quantized dynamics on the graph in Figure 4(b).
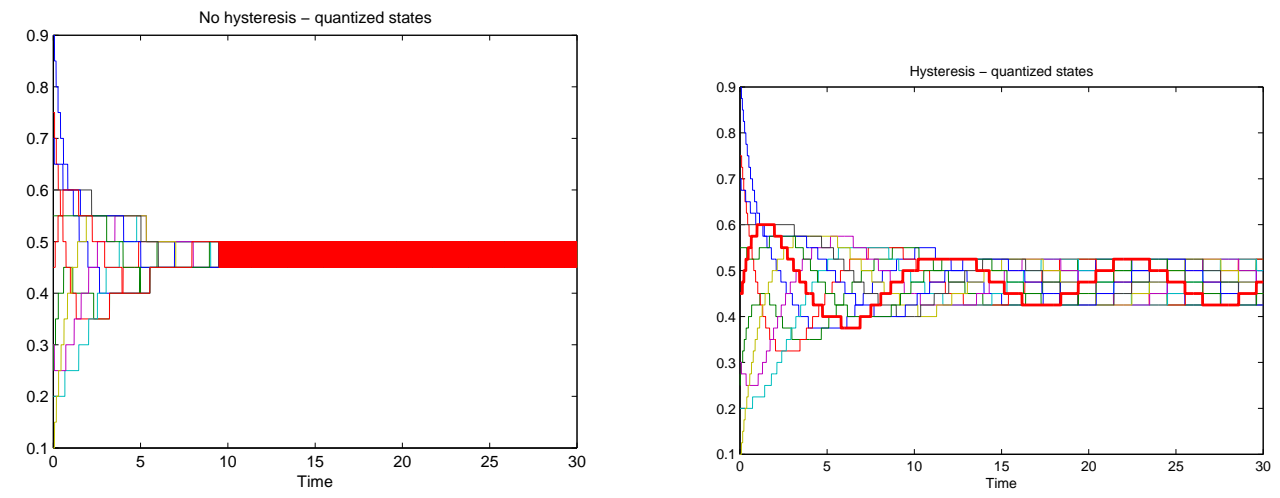

Figure 7: Examples of evolutions of quantized dynamics on a directed ring.

\section{References}

[1] J. P. Aubin and A. Cellina. Differential inclusions, volume 264 of Grundlehren der Mathematischen Wissenschaften. Springer, Berlin, 1984.

[2] T. C. Aysal, M. J. Coates, and M. G. Rabbat. Distributed average consensus with dithered quantization. IEEE Transactions on Signal Processing, 56(10):4905-4918, 2008.

[3] A. Bacciotti and F. Ceragioli. Stability and stabilization of discontinuous systems and nonsmooth Liapunov functions. ESAIM: Control, Optimisation 8 Calculus of Variations, 4:361-376, 1999.

[4] F. Bullo, J. Cortés, and S. Martínez. Distributed Control of Robotic Networks. Applied Mathematics Series. Princeton University Press, 2009.

[5] R. Carli, F. Bullo, and S. Zampieri. Quantized average consensus via dynamic coding/decoding schemes. International Journal of Robust and Nonlinear Control, 20(2):156-175, 2010. 
[6] R. Carli, F. Fagnani, P. Frasca, and S. Zampieri. Gossip consensus algorithms via quantized communication. Automatica, 46(1):70-80, 2010.

[7] J. Cortés. Finite-time convergent gradient flows with applications to network consensus. Automatica, 42(11):1993-2000, 2006.

[8] J. Cortés. Discontinuous dynamical systems - a tutorial on solutions, nonsmooth analysis, and stability. IEEE Control Systems Magazine, 28(3):36-73, 2008.

[9] J. Cortés. Distributed algorithms for reaching consensus on general functions. Automatica, 44(3):726-737, 2008.

[10] K. Deimling. Multivalued Differential Equations. De Gruyter, Berlin, 1992.

[11] D. V. Dimarogonas and K. H. Johansson. Stability analysis for multi-agent systems using the incidence matrix: Quantized communication and formation control. automatica, 46(4):695-700, 2010.

[12] A. F. Filippov. Differential Equations with Discontinuous Righthand Sides, volume 18 of Mathematics and Its Applications. Kluwer Academic Publishers, 1988.

[13] P. Frasca, R. Carli, F. Fagnani, and S. Zampieri. Average consensus on networks with quantized communication. International Journal of Robust and Nonlinear Control, 19(16):1787-1816, 2009.

[14] R. Goebel, R. G. Sanfelice, and A. R. Teel. Hybrid dynamical systems. IEEE Control Systems Magazine, 29(2):28-93, 2009.

[15] O. Hájek. Discontinuous differential equations I. Journal of Differential Equations, 32:149-170, 1979.

[16] A. Kashyap, T. Başar, and R. Srikant. Quantized consensus. Automatica, 43(7):1192$1203,2007$.

[17] T. Li, M. Fu, L. Xie, and J.-F. Zhang. Distributed consensus with limited communication data rate. IEEE Transactions on Automatic Control, 56(2):279-292, 2011.

[18] A. Nedic, A. Olshevsky, A. Ozdaglar, and J. N. Tsitsiklis. On distributed averaging algorithms and quantization effects. IEEE Transactions on Automatic Control, 54(11):2506-2517, 2009 .

[19] V. V. Nemytskii and V. V. Stepanov. Qualitative Theory of Differential Equations. Dover, New York, 1960.

[20] B. Paden and S. S. Sastry. A calculus for computing Filippov's differential inclusion with application to the variable structure control of robot manipulators. IEEE Transactions on Circuits and Systems, 34(1):73-82, 1987.

[21] M. Penrose. Random Geometric Graphs. Oxford Studies in Probability. Oxford University Press, 2003.

[22] J. Yu, S. M. LaValle, and D. Liberzon. Rendezvous without coordinates. In IEEE Conf. on Decision and Control, pages 1803-1808, Cancún, México, December 2008. 